\title{
Boundary Controllability Of Two Vibrating Strings Connected By A Point Mass With Variable Coefficients
}

\author{
Jamel Ben Amara * $\quad$ Emna Beldi ${ }^{\dagger}$
}

\begin{abstract}
S. Hansen and E. Zuazua [SIAM J. Cont. Optim., 1995] studied the problem of exact controllability of two strings connected by a point mass with constant physical coefficients. In this paper we study the same problem with variable physical coefficients. This system is generated by the following equations

$$
\begin{gathered}
\rho(x) u_{t t}=\left(\sigma(x) u_{x}\right)_{x}-q(x) u, \quad x \in(-1,0) \cup(0,1), t>0, \\
M u_{t t}(0, t)+\sigma_{1}(0) u_{x}\left(0^{-}, t\right)-\sigma_{2}(0) u_{x}\left(0^{+}, t\right)=0, \quad t>0,
\end{gathered}
$$

with Dirichlet boundary condition on the left end and a control acts on the right end. We prove that this system is exactly controllable in an asymmetric space for the control time $T>2 \int_{-1}^{1}\left(\frac{\rho(x)}{\sigma(x)}\right)^{\frac{1}{2}} d x$. We establish the equivalence between a suitable asymmetric norm of the initial data and the $L^{2}(0, T)$-norm of $u_{x}(1, t)$ (where $u$ is the solution of the uncontrolled system). Our approach is mainly based on a detailed spectral analysis and the theory of divided differences. More precisely, we prove that the spectral gap tends to zero with a precise asymptotic estimate.
\end{abstract}

Key words. Boundary control, point mass, Riesz basis, vibrating string, variable coefficients.

Mathematics Subject Classification. 35P, 47A, 93B.

\section{Introduction}

The controllability of mechanical structures with attached masses has been extensively investigated for several decades. To mention some examples, see [15] for heat equation connected by a point mass, [7, 14, 21] for strings with an interior point mass and [8, 9, 10] for beams with an attached point mass. See also [11, 19, 20] for networks of strings or beams with attached masses.

*Department of Mathematics, Faculty of Sciences of Tunis, University of Tunis el Manar, Mathematical Engineering Laboratory, Tunisia; e-mail: jamel.benamara@fsb.rnu.tn.

${ }^{\dagger}$ Department of Mathematics, Tunisia Polytechnic School, Mathematical Engineering Laboratory, University of Carthage, Tunisia; e-mail: em.beldi@gmail.com. 
In this paper we study the boundary controllability of a linear hybrid system modeling two vibrating non-homogeneous strings connected by a point mass. By means of the functions:

$$
\begin{gathered}
u=u(x, t), x \in(-1,0), t>0, \\
v=v(x, t), x \in(0,1), t>0,
\end{gathered}
$$

we describe the vertical displacements of the first and the second string, respectively. The position of the mass $M>0$ attached to the strings at the point $x=0$ is described by the function $z=z(t)$ for $t>0$.

The linear system modeling the vibrations of these strings is given as follows

$$
\begin{cases}\rho_{1}(x) u_{t t}=\left(\sigma_{1}(x) u_{x}\right)_{x}-q_{1}(x) u, & x \in(-1,0), t>0, \\ \rho_{2}(x) v_{t t}=\left(\sigma_{2}(x) v_{x}\right)_{x}-q_{2}(x) v, & x \in(0,1), t>0, \\ u(0, t)=v(0, t)=z(t), & t>0, \\ M z_{t t}(t)+\sigma_{1}(0) u_{x}(0, t)-\sigma_{2}(0) v_{x}(0, t)=0, & t>0,\end{cases}
$$

with the following boundary conditions

$$
u(-1, t)=v(1, t)=0, \text { for } t>0
$$

and the following initial conditions

$$
\begin{cases}u(x, 0)=u^{0}(x), u_{t}(x, 0)=u^{1}(x), & x \in(-1,0), \\ v(x, 0)=v^{0}(x), v_{t}(x, 0)=v^{1}(x), & x \in(0,1), \\ z(0)=z^{0}, z_{t}(0)=z^{1} & \end{cases}
$$

The coefficients $\rho_{i}(x)$ and $\sigma_{i}(x)$ represent respectively the density and the tension of each string, $i=1,2$. The potentials are denoted by the functions $q_{1}(x)$ and $q_{2}(x)$. In this study we are interested in the boundary controllability when a control function $p=p(t)$ acts on the system through the extreme $x=1$. Then the boundary conditions (1.2) is replaced by

$$
u(-1, t)=0, v(1, t)=p(t), \quad t>0 .
$$

Throughout this paper the coefficients $\rho_{i}$ and $\sigma_{i}$ are assumed to be uniformly positive, $q_{i}$ is nonnegative $(\mathrm{i}=1,2)$ and

$$
\begin{gathered}
\rho_{1}, \sigma_{1} \in H^{2}(-1,0), q_{1} \in L^{1}(-1,0), \\
\rho_{2}, \sigma_{2} \in H^{2}(0,1), q_{2} \in L^{1}(0,1) .
\end{gathered}
$$

In what follows, we introduce the following spaces

$$
\begin{aligned}
& \mathcal{H}_{0}=L^{2}(-1,0) \times L^{2}(0,1) \times \mathbb{R} \\
& \mathcal{V}_{1}=\left\{u \in H^{1}(-1,0) \mid u(-1)=0\right\} \\
& \mathcal{V}_{2}=\left\{v \in H^{1}(0,1) \mid v(1)=0\right\} \\
& \mathcal{V}=\left\{(u, v) \in \mathcal{V}_{1} \times \mathcal{V}_{2} \mid u(0)=v(0)\right\}
\end{aligned}
$$


and

$$
\mathcal{H}_{-1}=\mathcal{V}_{1}^{\prime} \times \mathcal{V}_{2}^{\prime} \times \mathbb{R}
$$

where the space $\left(\mathcal{V}_{i}\right)^{\prime}$ denotes the dual space of $\mathcal{V}_{i},(i=1,2)$.

In a similar way as in [14], we have the following existence and uniqueness result for System (1.1) $-(1.3)-(1.4)$ :

Proposition 1.1 For every $p \in L^{2}(0, T),\left(u^{0}, v^{0}, z^{0}\right) \in \mathcal{H}_{0}$ and $\left(u^{1}, v^{1}, z^{1}\right) \in \mathcal{H}_{-1}$ such that $\left(u^{0}, u^{1}\right) \in \mathcal{V}_{1} \times L^{2}(-1,0)$ and $u^{0}(0)=z^{0}$, there exists a solution of System (1.1) -(1.4) with initial data (1.3) in the class:

$$
(u, v, z) \in C\left([0, T], \mathcal{H}_{0}\right) \cap C^{1}\left([0, T], \mathcal{H}_{-1}\right)
$$

and

$$
u \in C\left([0, T], \mathcal{V}_{1}\right) \cap C^{1}\left([0, T], L^{2}(-1,0)\right) .
$$

Now, we can state our main result in this paper:

Theorem 1.2 Suppose

$$
T>2\left(\gamma_{1}+\gamma_{2}\right)=2\left(\int_{-1}^{0}\left(\frac{\rho_{1}(x)}{\sigma_{1}(x)}\right)^{\frac{1}{2}} d x+\int_{0}^{1}\left(\frac{\rho_{2}(x)}{\sigma_{2}(x)}\right)^{\frac{1}{2}} d x\right) .
$$

Then, for every $\left(u^{0}, v^{0}, z^{0}\right) \in \mathcal{H}_{0}$ and $\left(u^{1}, v^{1}, z^{1}\right) \in \mathcal{H}_{-1}$ such that $\left(u^{0}, u^{1}\right) \in \mathcal{V}_{1} \times L^{2}(-1,0)$ and $u^{0}(0)=z^{0}$, there exists a control $p \in L^{2}(0, T)$ such that the solution of (1.1) $-(1.3)-(1.4)$ satisfies

$$
\left\{\begin{array}{l}
u(x, T)=u_{t}(x, T)=0 \\
v(x, T)=v_{t}(x, T)=0 \\
z(T)=z_{t}(T)=0
\end{array}\right.
$$

For the proof of this theorem, we establish the following result: if $T>2\left(\gamma_{1}+\gamma_{2}\right)$, then for every solution $(u, v, z)$ of the uncontrolled problem (1.1)-(1.2)-(1.3) there exist two positive constants $C_{1}$ and $C_{2}$ such that

$$
C_{1}\left\|U^{0}\right\|_{\mathcal{Y}}^{2} \leq\left\|v_{x}(1, t)\right\|_{L^{2}(0, T)}^{2} \leq C_{2}\left\|U^{0}\right\|_{\mathcal{Y}}^{2}
$$

where $U^{0}=\left(u^{0}, v^{0}, z^{0}\right),\left(u^{1}, v^{1}, z^{1}\right)$ and

$$
\mathcal{Y}=\left\{U^{0}=\left(\left(u^{0}, v^{0}, z^{0}\right),\left(u^{1}, v^{1}, z^{1}\right)\right) \in \mathcal{H}_{0} \times \mathcal{H}_{-1} \mid\left(v^{0}, v^{1}\right) \in \mathcal{V}_{2} \times L^{2}(0,1)\right\}
$$

In [14], S. Hansen and E. Zuazua studied System (1.1)-(1.2), for constant physical parameters $\rho_{i}>0, \sigma_{i}>0$ and $q_{i} \equiv 0,(i=1,2)$ when the two strings occupy the intervals $\left(-\ell_{1}, 0\right)$ and $\left(0, \ell_{2}\right)\left(\ell_{1}, \ell_{2}>0\right)$. Using the Hilbert Uniqueness Method "HUM" (cf. J.L. Lions [18]), they proved the exact controllability in an asymmetric space when the control acts at one end of the string-mass-system for a control 
time $T>2\left(\ell_{1} \sqrt{\frac{\rho_{1}}{\sigma_{1}}}+\ell_{2} \sqrt{\frac{\rho_{2}}{\sigma_{2}}}\right)$. In the particular case, when $\rho_{i}=\sigma_{i}=\ell_{i}=1$ and $q_{i} \equiv 0(\mathrm{i}=1,2)$, the proof of the exact controllability is mainly based on the theory of non-harmonic Fourier series. Since in this case the spectral gap tends to zero, they employed a result of D. Ullrich [22] to prove the observability inequality. At the end of their paper, they considered the case of variable coefficients $\rho_{i}, \sigma_{i}$ and $q_{i}(i=1,2)$, where Theorem 1.2 was enunciated in a form of a conjecture. Later C. Castro in [7] proved the same result by using a different approach, he showed that the solutions of the above system can be viewed as weak limits of solutions of a string equation with unit density on $(-1,1) \backslash(-\epsilon, \epsilon)$ and with density $\frac{1}{2 \epsilon}$ on $(-\epsilon, \epsilon)$. Recently, S. Avdonin and J. Edward in [2] have studied the exact controllability of a vibrating string with $N$ attached masses inside an interval $(0, \ell)$ with a Dirichlet control at one end. Their problem in the case of a string with a single attached mass, coincides with System (1.1)-(1.2) for $\rho_{i}=\sigma_{i}=1(\mathrm{i}=1,2)$. Using a different approach that used in [7, 14, they proved the exact controllability in an asymmetric space for $T \geq 2 \ell$. In particular, they established the equivalence between an asymmetric norm of the initial data and the $L^{2}(0, T)$-norm of the control $p(t)$.

Our approach is essentially based on a precise computation of the associated spectral gap together with a suitable non-harmonic Fourier series result for the time exponentials. We show that there exists a subsequence of eigenfrequencies $\left(\sqrt{\lambda_{\varphi(n)}}\right)$ associated with System (1.1)-(1.2) such that $\sqrt{\lambda_{\varphi(n)+1}}-\sqrt{\lambda_{\varphi(n)}}=\mathcal{O}\left(\frac{1}{n}\right)$ and

$\sqrt{\lambda_{n+2}}-\sqrt{\lambda_{n}}>2 \delta, n \geq 1$, for some $\delta>0$. In the process of the last result, we establish an interpolation formula between the eigenvalues of System (1.1)-(1.2) and those of the regular problem (1.1)-(1.2) for $M=0$. Instead of Ullrich's theorem used in [7, 14], we here apply a result of C. Baiocchi et al. [5]. More general results concerning divided differences theory can be found in [3] and [4].

The rest of the paper is divided in the following way: In section 2 we associate to System (1.1)-(1.2) a self-adjoint operator defined in the Hilbert space $\mathcal{H}_{0}$ (defined by (1.5). Moreover, we give a result on the well-posedness of system (1.1)-(1.2)-(1.3). In the next section we establish the asymptotic properties of the associated spectral gap and the asymptotes of the eigenfunctions. In section 4 we define an asymmetric space and we give its characterization. In the last section we prove Theorem 1.2 and the observability inequality (1.8).

\section{Operator Framework And Well-posedness}

We begin with considering the following closed subspace of $\mathcal{V} \times \mathbb{R}$, where $\mathcal{V}$ is defined by (1.6)

$$
\mathcal{W}=\{(u, v, z) \in \mathcal{V} \times \mathbb{R}: u(0)=v(0)=z\}
$$

equipped with the norm

$$
\|(u, v, z)\|_{\mathcal{W}}^{2}=\|u\|_{\mathcal{V}_{1}}^{2}+\|v\|_{\mathcal{V}_{2}}^{2}
$$


where

$$
\|u\|_{\mathcal{V}_{1}}^{2}=\int_{-1}^{0}\left|u_{x}(x)\right|^{2} d x, \quad\|v\|_{\mathcal{V}_{2}}^{2}=\int_{0}^{1}\left|v_{x}(x)\right|^{2} d x
$$

and the spaces $\mathcal{V}_{1}$ and $\mathcal{V}_{2}$ are defined by (1.6).

In the sequel we introduce the operator $\mathcal{A}$ defined in $\mathcal{H}_{0}$, which is defined by (1.5), by setting:

$$
\mathcal{A} Y=\left\{\begin{array}{l}
\frac{1}{\rho_{1}}\left(-\left(\sigma_{1} u^{\prime}\right)^{\prime}+q_{1} u\right), \\
\frac{1}{\rho_{2}}\left(-\left(\sigma_{2} v^{\prime}\right)^{\prime}+q_{2} v\right), \\
\frac{1}{M}\left(\sigma_{1}(0) u^{\prime}(0)-\sigma_{2}(0) v^{\prime}(0)\right),
\end{array}\right.
$$

where $Y=(u, v, z)^{t}$ on the domain

$$
D(\mathcal{A})=\left\{Y \mid Y=(u, v, z) \in \mathcal{W}:(u, v) \in H^{2}(-1,0) \times H^{2}(0,1)\right\},
$$

which is dense in $\mathcal{H}_{0}$. Note that the space $\mathcal{H}_{0}$ is equipped with the scalar product $\langle. / .\rangle_{\mathcal{H}_{0}}$ defined by: for all $Y_{1}=\left(u_{1}, v_{1}, \alpha_{1}\right)^{t}$ and $Y_{2}=\left(u_{2}, v_{2}, \alpha_{2}\right)^{t} \in \mathcal{H}_{0}$, where ${ }^{t}$ denotes the transposition, we have

$$
\left\langle Y_{1}, Y_{2}\right\rangle_{\mathcal{H}_{0}}=\int_{-1}^{0} u_{1}(x) u_{2}(x) \rho_{1}(x) d x+\int_{0}^{1} v_{1}(x) v_{2}(x) \rho_{2}(x) d x+M \alpha_{1} \alpha_{2}
$$

Proposition 2.1 The linear operator $\mathcal{A}$ is self-adjoint and positive such that $\mathcal{A}^{-1}$ is compact. Moreover, the operator $\mathcal{A}^{\frac{1}{2}}$ generates a strongly continuous semi-group on $\mathcal{H}_{0}$.

Proof. Let $Y=(u, v, z)^{t} \in D(\mathcal{A})$, then by a simple integration by parts, we have

$$
\langle\mathcal{A} Y, Y\rangle_{\mathcal{H}_{0}}=\int_{-1}^{0} \sigma_{1}(x)\left|u^{\prime}\right|^{2} d x+\int_{-1}^{0} q_{1}(x)|u|^{2} d x+\int_{0}^{1} \sigma_{2}(x)\left|v^{\prime}\right|^{2} d x+\int_{0}^{1} q_{2}(x)|v|^{2} d x .
$$

Since $\sigma_{i}>0$ and $q_{i}>0,(\mathrm{i}=1,2)$, then $\langle\mathcal{A} Y, Y\rangle_{\mathcal{H}_{0}}>0$, and hence, the operator $\mathcal{A}$ is symmetric on $\mathcal{H}_{0}$. To prove that this operator is self-adjoint, it suffices to show that $\operatorname{Ran}(\mathcal{A}-i I d)=\mathcal{H}_{0}$, and this is omitted here.

It is easy to see that the space $\mathcal{W}$ is continuously and compactly embedded in the space $\mathcal{H}_{0}$, and hence, the operator $\mathcal{A}^{-1}$ is compact in $\mathcal{H}_{0}$.

According to Proposition 2.1, the operator $\mathcal{A}$ is positive and self-adjoint, and hence, it generates a scale of Hilbert spaces $\mathcal{H}_{\theta}=D\left(\mathcal{A}^{\theta}\right), \theta \in \mathbb{R}$. In particular, $\mathcal{H}_{0}$ is defined by (1.5) and $\mathcal{H}_{\frac{1}{2}}=D\left(\mathcal{A}^{\frac{1}{2}}\right)$. The norm in $\mathcal{H}_{\frac{1}{2}}$ is given by

$$
\begin{aligned}
\|y\|_{\frac{1}{2}}^{2} & =\left\langle\mathcal{A}^{\frac{1}{2}} y, \mathcal{A}^{\frac{1}{2}} y\right\rangle_{\mathcal{H}_{0}}, \\
& =\int_{-1}^{0}\left(\sigma_{1}(x)\left|u^{\prime}\right|^{2}+q_{1}(x)|u|^{2}\right) d x+\int_{0}^{1}\left(\sigma_{2}(x)\left|v^{\prime}\right|^{2}+q_{2}(x)|v|^{2}\right) d x .
\end{aligned}
$$

Our assumptions on $\sigma_{i}(x)$ and $q_{i}(x)(i=1,2)$ imply that $\mathcal{H}_{\frac{1}{2}}$ is topologically equivalent to the subspace $\mathcal{W}$. 
Proposition 2.2 The map $Y=(u, v, z) \rightarrow(u, v)$ is an homeomorphism from $D\left(\mathcal{A}^{\frac{1}{2}}\right)$ into $\mathcal{V}$, where $\mathcal{V}$ is defined by (1.6).

Obviously, the Cauchy problem (1.1) $-(1.3)$ can be rewritten in the abstract form

$$
\ddot{Y}=-\mathcal{A} Y,\left(Y(0), Y_{t}(0)\right)=\left(Y^{0}, Y^{1}\right)
$$

where $\mathcal{A}$ is defined by (2.4), $Y^{0}=\left(u^{0}, v^{0}, z^{0}\right)^{t}$ and $Y^{1}=\left(u^{1}, v^{1}, z^{1}\right)^{t}$. As a consequence of Proposition 2.1, we have the following existence and uniqueness result for Problem (1.1)-(1.2)-(1.3):

Theorem 2.3 For every $\left(\left(u^{0}, v^{0}\right),\left(u^{1}, v^{1}, z^{1}\right)\right) \in \mathcal{V} \times \mathcal{H}_{0}$, there exists a unique solution of System (1.1)-(1.2) with initial conditions (1.3) in the class

$$
(u, v, z) \in C([0, T], \mathcal{W}) \cap C^{1}\left([0, T], \mathcal{H}_{0}\right) .
$$

\section{Spectral Gap And Asymptotic Of The Eigen- functions}

In this section we investigate the spectral properties of the operator $\mathcal{A}$, in particular we establish the asymptotic behavior of the spectral gap $\sqrt{\lambda_{n+1}}-\sqrt{\lambda_{n}}$. Similar asymptotic estimate in the case of constant coefficients was obtained in [7, 14]. We consider the following spectral problem which arises by applying separation of variables to System (1.1)-(1.2):

$$
\begin{cases}-\left(\sigma_{1}(x) u^{\prime}(x)\right)^{\prime}+q_{1}(x) u(x)=\lambda \rho_{1}(x) u(x), & x \in(-1,0), \\ -\left(\sigma_{2}(x) v^{\prime}(x)\right)^{\prime}+q_{2}(x) v(x)=\lambda \rho_{2}(x) v(x), & x \in(0,1), \\ u(-1)=v(1)=0, u(0)=v(0), & \\ \sigma_{1}(0) u^{\prime}(0)-\sigma_{2}(0) v^{\prime}(0)=\lambda M u(0) . & \end{cases}
$$

Lemma 3.1 The spectrum of the operator $\mathcal{A}$ is discrete. It consists of an increasing sequence of positive and simple eigenvalues $\left(\lambda_{n}\right)_{n \in \mathbb{N}^{*}}$ tending to $+\infty$ :

$$
0<\lambda_{1}<\lambda_{2}<\ldots \ldots<\lambda_{n}<\ldots \ldots \underset{n \rightarrow+\infty}{\longrightarrow}+\infty
$$

Moreover, the corresponding eigenfunctions $\Phi_{n}(x)=\left(\phi_{n}(x), \phi_{n}(0)\right)$ form an orthogonal basis in $\mathcal{H}_{0}$, with $\phi_{n}$ are the eigenfunctions of the eigenvalue problem (3.1).

Proof. Here we have only to prove the simplicity of the eigenvalues $\lambda_{n}$ for all $n \in \mathbb{N}^{*}$. Let $\tilde{u}(x, \lambda)$ and $\tilde{v}(x, \lambda)$ be the solutions of the initial value problems

$$
\left\{\begin{array}{l}
-\left(\sigma_{1}(x) u^{\prime}\right)^{\prime}+q_{1}(x) u=\lambda \rho_{1}(x) u, \quad x \in(-1,0) \\
u(-1)=0, u^{\prime}(-1)=1
\end{array}\right.
$$


and

$$
\left\{\begin{array}{l}
-\left(\sigma_{2}(x) v^{\prime}\right)^{\prime}+q_{2}(x) v=\lambda \rho_{2}(x) v, \quad x \in(0,1) \\
v(1)=0, v^{\prime}(1)=-1
\end{array}\right.
$$

respectively. Let $\lambda$ be an eigenvalue of the operator $\mathcal{A}$ and $E_{\lambda}$ be the corresponding eigenspace. For any eigenfunction $\phi(x, \lambda)$ of $E_{\lambda}, \phi(x, \lambda)$ can be written in the form

$$
\phi(x, \lambda)= \begin{cases}c_{1} \tilde{u}(x, \lambda), & -1 \leq x \leq 0 \\ c_{2} \tilde{v}(x, \lambda), & 0 \leq x \leq 1\end{cases}
$$

where $c_{1}$ and $c_{2}$ are two constants. The first condition at $x=0$ of Problem (3.1) is equivalent to

$$
c_{1} \tilde{u}(0, \lambda)=c_{2} \tilde{v}(0, \lambda) .
$$

If $\tilde{u}(0, \lambda) \neq 0$ and $\tilde{v}(0, \lambda) \neq 0$, then $c_{1}=c_{2} \frac{\tilde{v}(0, \lambda)}{\tilde{u}(0, \lambda)}$, and hence, $\operatorname{Dim}\left(E_{\lambda}\right)=1$.

If $\tilde{u}(0, \lambda)=0$ and $\tilde{v}(0, \lambda) \neq 0$ (or $\tilde{u}(0, \lambda) \neq 0$ and $\tilde{v}(0, \lambda)=0$ ), then

$$
\phi(x, \lambda)= \begin{cases}c_{1} \tilde{u}(x, \lambda), & -1 \leq x \leq 0 \\ 0, & 0 \leq x \leq 1\end{cases}
$$

Since $\tilde{u}^{\prime}(0, \lambda) \neq 0$ and $\tilde{v}^{\prime}(0, \lambda)=0$, then the last condition in Problem (3.1) is not satisfied, a contradiction.

Now, if $\tilde{u}(0, \lambda)=\tilde{v}(0, \lambda)=0$, then from the last condition in (3.1), we have

$$
c_{1} \sigma_{1}(0) \tilde{u}^{\prime}(0)-c_{2} \sigma_{2}(0) \tilde{v}^{\prime}(0)=0 .
$$

Since $\tilde{u}^{\prime}(0, \lambda) \neq 0$ and $\tilde{v}^{\prime}(0, \lambda) \neq 0$, then $c_{1}=c_{2} \frac{\sigma_{2}(0) \tilde{v}^{\prime}(0, \lambda)}{\sigma_{1}(0) \tilde{u}^{\prime}(0, \lambda)}$, whence $\operatorname{Dim}\left(E_{\lambda}\right)=1$.

Since the operator $\mathcal{A}$ is self-adjoint, then the algebraic multiplicity of $\lambda$ is equal to one.

Now, we enunciate our main result in this section:

Theorem 3.2 There exists a subsequence of eigenvalues $\left(\lambda_{\varphi(n)}\right)_{n \geq 1}$ which satisfies the asymptotic estimates:

$$
\begin{aligned}
& \sqrt{\lambda_{\varphi(n)+1}}-\sqrt{\lambda_{\varphi(n)}}=\mathcal{O}\left(\frac{1}{n}\right), \\
& \sqrt{\lambda_{n+2}}-\sqrt{\lambda_{n}}>2 \delta, n \geq 1, \text { for some } \delta>0 .
\end{aligned}
$$

In order to prove this theorem, we establish some preliminary results. Let $\Gamma=\left\{\mu_{n}\right\}_{1}^{\infty}=\left\{\mu_{n}^{-}\right\}_{1}^{\infty} \bigcup\left\{\mu_{n}^{+}\right\}_{1}^{\infty}$, where $\mu_{n}^{-}$and $\mu_{n}^{+}$are the eigenvalues of the problems

$$
\left\{\begin{array}{l}
-\left(\sigma_{1}(x) y_{x}(x)\right)_{x}+q_{1}(x) y(x)=\lambda \rho_{1}(x) y(x), x \in(-1,0) \\
y(-1)=y(0)=0
\end{array}\right.
$$

and

$$
\left\{\begin{array}{l}
-\left(\sigma_{2}(x) y_{x}(x)\right)_{x}+q_{2}(x) y(x)=\lambda \rho_{2}(x) y(x), x \in(0,1) \\
y(0)=y(1)=0
\end{array}\right.
$$


respectively. It is known [17, Chapter 1], that the eigenvalues $\mu_{j}^{-}$and $\mu_{k}^{+}$satisfy the asymptotic estimates

$$
\left\{\begin{array}{l}
\sqrt{\mu_{j}^{-}}=\frac{j \pi}{\gamma_{1}}+\mathcal{O}\left(\frac{1}{j}\right) \\
\sqrt{\mu_{k}^{+}}=\frac{k \pi}{\gamma_{2}}+\mathcal{O}\left(\frac{1}{k}\right)
\end{array}\right.
$$

where

$$
\gamma_{1}=\int_{-1}^{0} \sqrt{\frac{\rho_{1}(x)}{\sigma_{1}(x)}} d x \text { and } \gamma_{2}=\int_{0}^{1} \sqrt{\frac{\rho_{2}(x)}{\sigma_{2}(x)}} d x .
$$

Obliviously, $\mu_{j}^{-}$and $\mu_{k}^{+}$can coincide. Let

$$
\Gamma^{*}=\left\{\mu_{n} \in \Gamma \backslash \mu_{j}^{-}=\mu_{k}^{+}, \text {for some } j, k \in \mathbb{N}^{*}\right\} .
$$

Note that if $\mu_{n} \in \Gamma^{*}$ (i.e., $\left.\tilde{u}\left(0, \mu_{n}\right)=\tilde{v}\left(0, \mu_{n}\right)=0\right)$, then $\mu_{n}$ is an eigenvalue of both problems (3.8) and (3.9). Clearly, all the eigenvalues $\mu_{n} \in \Gamma$ can be arranged as follows:

$$
0<\mu_{1} \leq \mu_{2} \leq \ldots \ldots . \leq \mu_{n} \leq \ldots \ldots \underset{n \rightarrow \infty}{\longrightarrow} \infty
$$

Remark 3.3 - If the coefficients $\rho_{i}, \sigma_{i}$ and $q_{i}(i=1,2)$ are symmetric, then $\Gamma \equiv \Gamma^{*}$.

- In the case of constant coefficients $\sigma_{i} \equiv 1, q_{i} \equiv 0(i=1,2)$, if $\sqrt{\frac{\rho_{1}}{\rho_{2}}} \in \mathbb{R} \backslash \mathbb{Q}$, then $\Gamma^{*} \equiv \emptyset$.

We consider the following boundary value problem

$$
\left\{\begin{array}{l}
-\left(\sigma_{1}(x) u_{x}\right)_{x}+q_{1}(x) u=\lambda \rho_{1}(x) u, \quad x \in(-1,0) \\
-\left(\sigma_{2}(x) v_{x}\right)_{x}+q_{2}(x) v=\lambda \rho_{2}(x) v, \quad x \in(0,1), \\
u(-1)=v(1)=0 \\
u(0)=v(0)
\end{array}\right.
$$

It is clear that for $\lambda \in \mathbb{C} \backslash \Gamma$, the set of solutions of Problem (3.13) is a one-dimensional subspace which is generated by a solution of the form

$$
\widetilde{U}(x, \lambda)=\left\{\begin{array}{l}
\tilde{v}(0, \lambda) \tilde{u}(x, \lambda), \quad-1 \leq x \leq 0 \\
\tilde{u}(0, \lambda) \tilde{v}(x, \lambda), \quad 0 \leq x \leq 1
\end{array}\right.
$$

where $\tilde{u}(x, \lambda)$ and $\tilde{v}(x, \lambda)$ are the solutions of the initial value problems (3.2) and (3.3), respectively. Note that $\tilde{u}(0, \lambda) \neq 0$ and $\tilde{v}(0, \lambda) \neq 0$ for $\lambda \in\left(\mu_{n}, \mu_{n+1}\right)$, since otherwise $\lambda$ would be an eigenvalue of Problem (3.8) or (3.9). Let us introduce the variable complex function

$$
F(\lambda)=\frac{\sigma_{1}(0) \widetilde{U}_{x}\left(0^{-}, \lambda\right)-\sigma_{2}(0) \widetilde{U}_{x}\left(0^{+}, \lambda\right)}{\widetilde{U}(0, \lambda)}, \lambda \in \mathbb{C} \backslash \Gamma,
$$


which can be rewritten in the form

$$
F(\lambda)=\frac{\sigma_{1}(0) \tilde{v}(0) \tilde{u}_{x}\left(0^{-}\right)-\sigma_{2}(0) \tilde{u}(0) \tilde{v}_{x}\left(0^{+}\right)}{\tilde{u}(0) \tilde{v}(0)}, \lambda \in \mathbb{C} \backslash \Gamma .
$$

It is known [17, Chapter 1], that $\tilde{u}(x, \lambda)$ and $\tilde{v}(x, \lambda)$ are entire functions in $\lambda$ and continuous on the intervals $[-1,0]$ and $[0,1]$, respectively. Therefore, $F(\lambda)$ is a meromorphic function. We will show below that its zeros and poles coincide with the eigenvalues of the regular problem (3.1) for $M=0$ and the eigenvalues $\mu_{n}, n \geq 1$, respectively. Moreover, the solution of the equation

$$
F(\lambda)=M \lambda,
$$

are the eigenvalues $\lambda_{n}, n \geq 1$ of Problem (3.1).

Lemma 3.4 The function $F(\lambda)$ is decreasing along the intervals $\left(-\infty, \mu_{1}\right)$ and $\left(\mu_{n}, \mu_{n+1}\right), n \geq 1$ with $\mu_{n} \neq \mu_{n+1}$. Furthermore, it decreases from $+\infty$ to $-\infty$ in all intervals.

Proof. Let $\left(\lambda, \lambda^{\prime}\right) \in\left(\mu_{n}, \mu_{n+1}\right) \times\left(\mu_{n}, \mu_{n+1}\right)$, where $\lambda \neq \lambda^{\prime}$ and $\widetilde{U}(x, \lambda), \widetilde{U}\left(x, \lambda^{\prime}\right)$ be the solutions of Problem (3.13). Integrating by parts and taking into account the boundary conditions in (3.13), yield

$$
\left\{\begin{array}{l}
\int_{-1}^{0}\left(\left(q_{1}-\lambda \rho_{1}\right)(x) \tilde{u}(x, \lambda) \tilde{u}\left(x, \lambda^{\prime}\right)+\sigma_{1}(x) \tilde{u}_{x}(x, \lambda) \tilde{u}_{x}\left(x, \lambda^{\prime}\right)\right) d x=\sigma_{1}(0) \tilde{u}\left(0, \lambda^{\prime}\right) \tilde{u}_{x}\left(0^{-}, \lambda\right) \\
\int_{-1}^{0}\left(\left(q_{1}-\lambda^{\prime} \rho_{1}\right)(x) \tilde{u}\left(x, \lambda^{\prime}\right) \tilde{u}(x, \lambda)+\sigma_{1}(x) \tilde{u}_{x}\left(x, \lambda^{\prime}\right) \tilde{u}_{x}(x, \lambda)\right) d x=\sigma_{1}(0) \tilde{u}(0, \lambda) \tilde{u}_{x}\left(0^{-}, \lambda^{\prime}\right)
\end{array}\right.
$$

and

$$
\left\{\begin{array}{l}
\int_{0}^{1}\left(\left(q_{2}-\lambda \rho_{2}\right)(x) \tilde{v}(x, \lambda) \tilde{v}\left(x, \lambda^{\prime}\right)+\sigma_{2}(x) \tilde{v}_{x}(x, \lambda) \tilde{v}_{x}\left(x, \lambda^{\prime}\right)\right) d x=-\sigma_{2}(0) \tilde{v}\left(0, \lambda^{\prime}\right) \tilde{v}_{x}\left(0^{+}, \lambda\right) \\
\int_{0}^{1}\left(\left(q_{2}-\lambda^{\prime} \rho_{2}\right)(x) \tilde{v}\left(x, \lambda^{\prime}\right) \tilde{v}(x, \lambda)+\sigma_{2}(x) \tilde{v}_{x}\left(x, \lambda^{\prime}\right) \tilde{v}_{x}(x, \lambda)\right) d x=-\sigma_{2}(0) \tilde{v}(0, \lambda) \tilde{v}_{x}\left(0^{+}, \lambda^{\prime}\right) .
\end{array}\right.
$$

Subtracting the two equations of Systems (3.17) and (3.18), one gets

$$
\left\{\begin{array}{l}
\left(\lambda^{\prime}-\lambda\right) \int_{-1}^{0} \rho_{1}(x) \tilde{u}(x, \lambda) \tilde{u}\left(x, \lambda^{\prime}\right) d x=\sigma_{1}(0)\left(\tilde{u}\left(0, \lambda^{\prime}\right) \tilde{u}_{x}\left(0^{-}, \lambda\right)-\tilde{u}(0, \lambda) \tilde{u}_{x}\left(0^{-}, \lambda^{\prime}\right)\right) \\
\left(\lambda^{\prime}-\lambda\right) \int_{0}^{1} \rho_{2}(x) \tilde{v}(x, \lambda) \tilde{v}\left(x, \lambda^{\prime}\right) d x=\sigma_{2}(0)\left(\tilde{v}(0, \lambda) \tilde{v}_{x}\left(0^{+}, \lambda^{\prime}\right)-\tilde{v}\left(0, \lambda^{\prime}\right) \tilde{v}_{x}\left(0^{+}, \lambda\right)\right)
\end{array}\right.
$$

Hence

$$
\begin{aligned}
\left(\lambda-\lambda^{\prime}\right) \int_{-1}^{0} \rho_{1}(x) \tilde{u}(x, \lambda) \tilde{u}\left(x, \lambda^{\prime}\right) d x= & \sigma_{1}(0)\left(\tilde{u}(0, \lambda)\left(\tilde{u}_{x}\left(0^{-}, \lambda^{\prime}\right)-\tilde{u}_{x}\left(0^{-}, \lambda\right)\right)\right. \\
& -\sigma_{1}(0) \tilde{u}_{x}\left(0^{-}, \lambda\right)\left(\tilde{u}\left(0, \lambda^{\prime}\right)-\tilde{u}(0, \lambda)\right)
\end{aligned}
$$


and

$$
\begin{aligned}
\left(\lambda^{\prime}-\lambda\right) \int_{0}^{1} \rho_{2}(x) \tilde{v}(x, \lambda) \tilde{v}\left(x, \lambda^{\prime}\right) d x= & \sigma_{2}(0)\left(\tilde{v}(0, \lambda)\left(\tilde{v}_{x}\left(0^{+}, \lambda^{\prime}\right)-\tilde{v}_{x}\left(0^{+}, \lambda\right)\right)\right. \\
& -\sigma_{2}(0) \tilde{v}_{x}\left(0^{+}, \lambda\right)\left(\tilde{v}\left(0, \lambda^{\prime}\right)-\tilde{v}(0, \lambda)\right) .
\end{aligned}
$$

Passing to the limit as $\lambda^{\prime} \rightarrow \lambda$, we get the identities

$$
\left\{\begin{array}{l}
-\int_{-1}^{0} \rho_{1}(x) \tilde{u}^{2}(x, \lambda) d x=\sigma_{1}(0)\left(\tilde{u}(0, \lambda) \frac{\partial \tilde{u}_{x}\left(0^{-}, \lambda\right)}{\partial \lambda}-\tilde{u}_{x}\left(0^{-}, \lambda\right) \frac{\partial \tilde{u}(0, \lambda)}{\partial \lambda}\right) \\
-\int_{0}^{1} \rho_{2}(x) \tilde{v}^{2}(x, \lambda) d x=-\sigma_{2}(0)\left(\tilde{v}(0, \lambda) \frac{\partial \tilde{v}_{x}\left(0^{+}, \lambda\right)}{\partial \lambda}-\tilde{v}_{x}\left(0^{+}, \lambda\right) \frac{\partial \tilde{v}(0, \lambda)}{\partial \lambda}\right) .
\end{array}\right.
$$

Dividing the first equation in (3.22) by $\tilde{u}^{2}(0, \lambda)$ and the second by $\tilde{v}^{2}(0, \lambda)$, it follows

$$
\frac{\partial F(\lambda)}{\partial \lambda}=-\frac{\tilde{v}^{2}(0, \lambda) \int_{-1}^{0} \rho_{1}(x) \tilde{u}^{2}(x, \lambda) d x+\tilde{u}^{2}(0, \lambda) \int_{0}^{1} \rho_{2}(x) \tilde{v}^{2}(x, \lambda) d x}{\tilde{u}^{2}(0, \lambda) \tilde{v}^{2}(0, \lambda)}<0 .
$$

In order to prove the second statement, we firstly establish the asymptotic of $F(\lambda)$ as $\lambda \rightarrow-\infty$. It is known (e.g; [12, Chapter 2] and [17, Chapter 1]) for $\lambda \in \mathbb{C}$ and $|\lambda| \rightarrow \infty$ that

$$
\left\{\begin{array}{l}
\tilde{u}(x, \lambda)=a_{1}\left(\rho_{1}(x) \sigma_{1}(x)\right)^{-\frac{1}{4}} \frac{\sin \left(\sqrt{\lambda} \int_{-1}^{x} \sqrt{\frac{\rho_{1}(t)}{\sigma_{1}(t)}}\right)}{\sqrt{\lambda}}[1] \\
\tilde{u}_{x}(x, \lambda)=a_{1}\left(\rho_{1}(x)\right)^{\frac{1}{4}}\left(\sigma_{1}(x)\right)^{-\frac{3}{4}} \cos \left(\sqrt{\lambda} \int_{-1}^{x} \sqrt{\frac{\rho_{1}(t)}{\sigma_{1}(t)}} d t\right)[1]
\end{array}\right.
$$

and

$$
\left\{\begin{array}{l}
\tilde{v}(x, \lambda)=a_{2}\left(\rho_{2}(x) \sigma_{2}(x)\right)^{-\frac{1}{4}} \frac{\sin \left(\sqrt{\lambda} \int_{x}^{1} \sqrt{\frac{\rho_{2}(t)}{\sigma_{2}(t)}} d t\right)}{\sqrt{\lambda}}[1] \\
\tilde{v}_{x}(x, \lambda)=-a_{2}\left(\rho_{2}(x)\right)^{\frac{1}{4}}\left(\sigma_{2}(x)\right)^{-\frac{3}{4}} \cos \left(\sqrt{\lambda} \int_{x}^{1} \sqrt{\frac{\rho_{2}(t)}{\sigma_{2}(t)}} d t\right)[1]
\end{array}\right.
$$

where $[1]=1+\mathcal{O}\left(\frac{1}{\sqrt{\lambda}}\right), \gamma_{i}(i=1,2)$ are defined by (3.11) and the constants

$$
\left\{\begin{array}{l}
a_{1}=\left(\left(\rho_{1}(-1)\right)^{\frac{1}{4}}\left(\sigma_{1}(-1)\right)^{-\frac{3}{4}}\right)^{-1} \\
a_{2}=\left(\left(\rho_{2}(1)\right)^{\frac{1}{4}}\left(\sigma_{2}(1)\right)^{-\frac{3}{4}}\right)^{-1}
\end{array}\right.
$$

are determined by the initial conditions in (3.2) and (3.3). By use of (3.24) and (3.25), a straightforward calculation gives the following asymptotic

$$
F(\lambda) \sim \sqrt{|\lambda|}\left(\sqrt{\rho_{1}(0) \sigma_{1}(0)}+\sqrt{\rho_{2}(0) \sigma_{2}(0)}\right) \text {, as } \lambda \rightarrow-\infty .
$$


This implies that $\lim _{\lambda \rightarrow-\infty} F(\lambda)=+\infty$.

Now, we prove that

$$
\lim _{\lambda \rightarrow \mu_{n}+0} F(\lambda)=+\infty, \quad \lim _{\lambda \rightarrow \mu_{n}-0} F(\lambda)=-\infty
$$

Suppose that $\tilde{u}\left(0, \mu_{n}\right)=\tilde{v}\left(0, \mu_{n}\right)=0$. Let $\lambda=\mu_{n}+\epsilon$, where $\epsilon$ is small enough. Therefore, a simple calculation yields

$$
\begin{aligned}
& F(\lambda)=\frac{\sigma_{1}(0) \tilde{u}_{x}\left(0, \mu_{n}\right) \frac{\partial \tilde{v}}{\partial \lambda}\left(0, \mu_{n}\right)-\sigma_{2}(0) \tilde{v}_{x}\left(0, \mu_{n}\right) \frac{\partial \tilde{u}}{\partial \lambda}\left(0, \mu_{n}\right)}{\epsilon \frac{\partial \tilde{u}}{\partial \lambda}\left(0, \mu_{n}\right) \frac{\partial \tilde{v}}{\partial \lambda}\left(0, \mu_{n}\right)}+\mathcal{O}(1), \\
&= \\
& \frac{1}{\epsilon}\left(\frac{\sigma_{1}(0) \tilde{u}_{x}\left(0, \mu_{n}\right)}{\frac{\partial \tilde{u}}{\partial \lambda}\left(0, \mu_{n}\right)}-\frac{\sigma_{2}(0) \tilde{v}_{x}\left(0, \mu_{n}\right)}{\frac{\partial \tilde{v}}{\partial \lambda}\left(0, \mu_{n}\right)}\right)+o(1) .
\end{aligned}
$$

Since $\mu_{n}$ is a simple eigenvalue of the two problems (3.8) and (3.9), then $\frac{\partial \tilde{u}}{\partial \lambda}\left(0, \mu_{n}\right) \neq 0$ and $\frac{\partial \tilde{v}}{\partial \lambda}\left(0, \mu_{n}\right) \neq 0$. Let us denote by

$$
F_{1}(\lambda)=\frac{\sigma_{1}(0) \tilde{u}_{x}(0, \lambda)}{\tilde{u}(0, \lambda)} \text { and } F_{2}(\lambda)=\frac{\sigma_{2}(0) \tilde{v}_{x}(0, \lambda)}{\tilde{v}(0, \lambda)} .
$$

It is easily seen that the eigenvalues $\mu_{j}^{-}$and $\mu_{k}^{+}(j \geq 1, k \geq 1)$ are the poles of $F_{1}(\lambda)$ and $F_{2}(\lambda)$, respectively. In view of the proof of Proposition 4 in [6], by use of the Mittag-Lefleur theorem [16, Chapter 4], $F_{1}(\lambda)$ has the following decomposition

$$
F_{1}(\lambda)=\sum_{j \geq 1}\left(\frac{\lambda}{\mu_{j}^{-}}\right) \frac{c_{j}}{\lambda-\mu_{j}^{-}},
$$

where $c_{j}$ are the residuals of $F_{1}(\lambda)$ at the poles $\mu_{j}^{-}, j \geq 1$. It is known that the residuals of $F_{1}(\lambda)$ and $F_{2}(\lambda)$ (at the poles $\mu_{j}^{-}$and $\mu_{k}^{+}$, respectively) are given by

$$
c_{j}=\frac{\sigma_{1}(0) \tilde{u}_{x}\left(0, \mu_{j}^{-}\right)}{\frac{\partial \tilde{u}}{\partial \lambda}\left(0, \mu_{j}^{-}\right)}, \quad c_{k}^{\prime}=\frac{\sigma_{2}(0) \tilde{v}_{x}\left(0, \mu_{k}^{+}\right)}{\frac{\partial \tilde{v}}{\partial \lambda}\left(0, \mu_{k}^{+}\right)} .
$$

According again to the proof of Proposition 4 in [6], we have $c_{j}>0, j \geq 1$. By the change of variables $s=-x$, if $\tilde{v}(x, \lambda)$ is a solution of Problem (3.3), then $\tilde{v}(-s, \lambda)$ is a solution of Problem (3.2) (where the coefficients are replaced by $\rho_{2}(-s), \sigma_{2}(-s)$ and $\left.q_{2}(-s)\right)$, and hence, $F_{2}(\lambda)=-\frac{\sigma_{2}(0) \tilde{s}_{s}(0, \lambda)}{\tilde{v}(0, \lambda)}$. Therefore, $c_{k}^{\prime}<0$ for all $k \geq 1$ and

$$
\frac{\sigma_{1}(0) \tilde{u}_{x}\left(0, \mu_{n}\right)}{\frac{\partial \tilde{u}}{\partial \lambda}\left(0, \mu_{n}\right)}-\frac{\sigma_{2}(0) \tilde{v}_{x}\left(0, \mu_{n}\right)}{\frac{\partial \tilde{v}}{\partial \lambda}\left(0, \mu_{n}\right)}>0, n \geq 1 .
$$

Passing to the limit as $\epsilon \rightarrow 0$ in (3.28), we obtain the first limit in (3.27). Analogously, we can prove the second limit of (3.27).

Now, if $\tilde{u}\left(0, \mu_{n}\right)=0$ and $\tilde{v}\left(0, \mu_{n}\right) \neq 0\left(\right.$ or $\tilde{v}\left(0, \mu_{n}\right)=0$ and $\left.\tilde{u}\left(0, \mu_{n}\right) \neq 0\right)$, then 
$\sigma_{1}(0) \tilde{u}_{x}\left(0, \mu_{n}\right) \neq 0\left(\right.$ or $\left.\sigma_{2}(0) \tilde{v}_{x}\left(0, \mu_{n}\right) \neq 0\right)$, and hence, we arrive to the same conclusion.

From the last lemma, it is clear that the poles of $F(\lambda)$ coincide with the eigenvalues $\mu_{n}, n \geq 1$. Furthermore, it follows the following interpolation formulas between the eigenvalues $\lambda_{n}, \mu_{n}$ and those of the regular problem (3.1) for $M=0$.

Proposition 3.5 Let $\lambda_{n}^{\prime}, n \geq 1$ denote the eigenvalues of the regular problem (3.1) for $M=0$. If $\mu_{n} \neq \mu_{n+1}$, then

$$
\lambda_{1}<\lambda_{1}^{\prime}<\mu_{1} \text { and } \mu_{n}<\lambda_{n+1}<\lambda_{n+1}^{\prime}<\mu_{n+1}, n \geq 1
$$

and if $\mu_{n}=\mu_{n+1}$, then $\mu_{n}=\lambda_{n+1}=\lambda_{n+1}^{\prime}$. Furthermore the eigenvalues $\left(\lambda_{n}\right)_{n \geq 1}$ satisfy the asymptotic

$$
\lambda_{n} \sim\left(\frac{n \pi}{\gamma_{1}+\gamma_{2}}\right)^{2}
$$

where $\gamma_{1}$ and $\gamma_{2}$ are defined by (3.11).

Proof. According to Lemma 3.4, $F(\lambda)$ is a decreasing function from $+\infty$ to $-\infty$ along each of intervals $\left(-\infty, \mu_{1}\right)$ and $\left(\mu_{n}, \mu_{n+1}\right), n \geq 1$. Hence, Equation (3.16) has exactly one zero in each of these intervals. Moreover, the equation $F(\lambda)=0$ has exactly one zero in each of these intervals. It is clear that these zeros (denoted by $\lambda_{n}^{\prime}$ ) are the eigenvalues of the regular problem (3.1) for $M=0$. Consequently, the interpolation formulas (3.30) are simple deductions from the graphs of the functions $F(\lambda)$ and $M \lambda$.

It is known (e.g., [1] and [13, Chapter 6.7]), that the eigenvalues $\lambda_{n}^{\prime}$ satisfy the asymptotes

$$
\lambda_{n}^{\prime}=\left(\frac{n \pi}{\int_{-1}^{1} \sqrt{\frac{\rho(x)}{\sigma(x)}} d x}\right)^{2}+\mathcal{O}(1)
$$

where

$$
\rho(x)=\left\{\begin{array}{ll}
\rho_{1}(x), & x \in[-1,0], \\
\rho_{2}(x), & x \in[0,1],
\end{array} \quad \text { and } \sigma(x)=\left\{\begin{array}{ll}
\sigma_{1}(x), & x \in[-1,0], \\
\sigma_{2}(x), & x \in[0,1],
\end{array} .\right.\right.
$$

Since $\lambda_{n-1}^{\prime}<\lambda_{n} \leq \lambda_{n}^{\prime}$, then (3.31) follows from (3.32).

Lemma 3.6 Let $\left(\mu_{n_{k}}\right)$ be a subsequence of $\left(\mu_{n}\right)$ such that

$$
\left|\sqrt{\mu_{n_{k}}}-\sqrt{\mu_{n_{k}-1}}\right| \rightarrow 0, \text { as } k \rightarrow \infty \text {. }
$$

(i) If $\mu_{n_{k}}$ is an eigenvalue of Problem (3.8) (or of Problem (3.9)), then $\mu_{n_{k}-1}$ is an eigenvalue of Problem (3.9) (or of Problem (3.8)).

Furthermore, $\sqrt{\mu_{n_{k}+1}}-\sqrt{\mu_{n_{k}}}>\delta_{1}$ and $\sqrt{\mu_{n_{k}-1}}-\sqrt{\mu_{n_{k}-2}}>\delta_{2}$ for some $\delta_{1}$, $\delta_{2}>0$. 
(ii) If for some $C>0$,

$$
\sqrt{\lambda_{n_{k}}}\left|\tilde{u}\left(0, \lambda_{n_{k}}\right)\right| \leq \frac{C}{k}\left(\text { or } \sqrt{\lambda_{n_{k}}}\left|\tilde{v}\left(0, \lambda_{n_{k}}\right)\right| \leq \frac{C}{k}\right) \text {, for large } k \in \mathbb{N}^{*},
$$

then

$$
\left|\tilde{u}\left(0, \lambda_{n_{k}}\right)\right| \sim\left|\tilde{v}\left(0, \lambda_{n_{k}}\right)\right| \text {, as } k \rightarrow \infty .
$$

\section{Proof.}

(i) It is clear from the asymptotes (3.10) and (3.33), that the eigenvalues $\mu_{n_{k}}$ and $\mu_{n_{k}-1}$ are of different types i.e., if $\mu_{n_{k}}$ is an eigenvalue of Problem (3.8), then $\mu_{n_{k}-1}$ is an eigenvalue of Problem (3.9)), or conversely.

Moreover, $\sqrt{\mu_{n_{k}+1}}-\sqrt{\mu_{n_{k}}}>\delta_{1}$ and $\sqrt{\mu_{n_{k}-1}}-\sqrt{\mu_{n_{k}-2}}>\delta_{2}$ for some $\delta_{1}, \delta_{2}>0$.

(ii) We may first assume for large $k \in \mathbb{N}^{*}$, that $\sqrt{\lambda_{n_{k}}}\left|\tilde{u}\left(0, \lambda_{n_{k}}\right)\right|<\frac{C}{k}, C>0$. Using the expression (3.15) of $F(\lambda)$ and the characteristic equation (3.16),

$$
\left|\frac{\tilde{v}\left(0, \lambda_{n_{k}}\right)}{\tilde{u}\left(0, \lambda_{n_{k}}\right)}\right|=\left|\frac{\sigma_{2}(0) \tilde{v}_{x}\left(0, \lambda_{n_{k}}\right)}{\sigma_{1}(0) \tilde{u}_{x}\left(0, \lambda_{n_{k}}\right)-M \lambda_{n_{k}} \tilde{u}\left(0, \lambda_{n_{k}}\right)}\right|,
$$

and hence, by (3.24) and (3.25), one has

$$
\left|\frac{\tilde{v}\left(0, \lambda_{n_{k}}\right)}{\tilde{u}\left(0, \lambda_{n_{k}}\right)}\right|=\left|\frac{a_{2}\left(\rho_{2}(0) \sigma_{2}(0)\right)^{\frac{1}{4}} \cos \left(\sqrt{\lambda_{n_{k}}} \gamma_{2}\right)[1]}{a_{1}\left(\left(\rho_{1}(0) \sigma_{1}(0)\right)^{\frac{1}{4}} \cos \left(\sqrt{\lambda_{n_{k}}} \gamma_{1}\right)-M \sqrt{\lambda_{n_{k}}}\left(\rho_{1}(0) \sigma_{1}(0)\right)^{\frac{-1}{4}} \sin \left(\sqrt{\lambda_{n_{k}}} \gamma_{1}\right)\right)[1]}\right|,
$$

the constants $a_{i}(i=1,2)$ are defined by $(\underline{3.26})$ and $[1]=1+\frac{1}{\sqrt{\lambda_{n_{k}}}}$. Under the above assumption together with (3.31),

$$
\sqrt{\lambda_{n_{k}}}\left|\sin \left(\sqrt{\lambda_{n_{k}}} \gamma_{1}\right)\right|[1] \rightarrow 0 \text { as } k \rightarrow \infty .
$$

In view of assertion (i), we may assume that $\mu_{n_{k}-1}$ is an eigenvalue of Problem (3.8) and $\mu_{n_{k}}$ is an eigenvalue of Problem (3.9) (the other case can be handled in a same way). We set

$$
\delta_{n_{k}}^{-}=\sqrt{\lambda_{n_{k}}}-\sqrt{\mu_{n_{k}-1}} \text { and } \delta_{n_{k}}^{+}=\sqrt{\mu_{n_{k}}}-\sqrt{\lambda_{n_{k}}} .
$$

Hence, by (3.30) and (3.33),

$$
\delta_{n_{k}}^{+} \rightarrow 0 \text { and } \delta_{n_{k}}^{-} \rightarrow 0, \text { as } k \rightarrow \infty .
$$

In view of (3.10), (3.39) and (3.40), we obtain

$$
\left|\sin \left(\sqrt{\lambda_{n_{k}}} \gamma_{2}\right)\right| \rightarrow 0, \text { as } k \rightarrow \infty .
$$


From this and (3.38), we have $\cos \left(\sqrt{\lambda_{n_{k}}} \gamma_{1}\right)=\mathcal{O}(1)$ and $\cos \left(\sqrt{\lambda_{n_{k}}} \gamma_{2}\right)=\mathcal{O}(1)$. Therefore, by (3.37),

$$
\left|\frac{\tilde{v}\left(0, \lambda_{n_{k}}\right)}{\tilde{u}\left(0, \lambda_{n_{k}}\right)}\right| \sim \frac{a_{2}}{a_{1}}\left(\frac{\rho_{2}(0) \sigma_{2}(0)}{\rho_{1}(0) \sigma_{1}(0)}\right)^{\frac{1}{4}}, \text { for } k \text { large enough. }
$$

Now, we assume for large $k \in \mathbb{N}^{*}$, that

$$
\sqrt{\lambda_{n_{k}}}\left|\tilde{u}\left(0, \lambda_{n_{k}}\right)\right|=\frac{C}{k}+o\left(\frac{1}{k}\right), C>0 .
$$

In the expression (3.36), suppose that

$$
\sigma_{1}(0) \tilde{u}_{x}\left(0, \lambda_{n_{k}}\right)-M \lambda_{n_{k}} \tilde{u}\left(0, \lambda_{n_{k}}\right) \rightarrow 0 \text {, as } k \rightarrow \infty .
$$

Substituting (3.24) and (3.41) into (3.42), one obtains

$$
M \sqrt{\lambda_{n_{k}}} \frac{C}{k}=\left(\rho_{1}(0) \sigma_{1}(0)\right)^{\frac{1}{2}}+\mathcal{O}\left(\frac{1}{k}\right) .
$$

Using this and (3.10) together with (3.39) and (3.40), one gets

$$
M \frac{C \pi}{\gamma_{1}}=\left(\rho_{1}(0) \sigma_{1}(0)\right)^{\frac{1}{2}} \text { and } M \frac{C \pi}{\gamma_{2}}=\left(\rho_{1}(0) \sigma_{1}(0)\right)^{\frac{1}{2}} .
$$

Then if $\gamma_{1} \neq \gamma_{2}$, this is in contradiction with (3.42). If $\gamma_{1}=\gamma_{2}$, it is easily seen from (3.24) and (3.25), that (3.35) holds. This completes the proof.

We are now ready to prove Theorem 3.2 .

Proof. First, we establish the asymptotic estimate

$$
\sqrt{\lambda_{n}}-\sqrt{\mu_{n-1}}=\mathcal{O}\left(\frac{1}{n}\right), \text { for } n \in \Omega
$$

where for some $\tau>0$,

$$
\Omega=\left\{n \in \mathbb{N}^{*}: \mu_{n}-\mu_{n-1} \geq \tau\right\} .
$$

First of all, let us recall from the asymptotics (3.10) that for large $n \in \Omega$, we have

$$
\sqrt{\mu_{n}}-\sqrt{\mu_{n-1}} \geq \frac{\tau_{0}}{n}
$$

for some $\tau_{0}>0$. For $\xi \in\left(\sqrt{\mu_{n-1}}, \sqrt{\lambda_{n}}\right]$ we put $G(\xi)=\frac{1}{F\left(\xi^{2}\right)}$, where $F$ is defined by (3.15). In view of Proposition [3.5, $\left(\sqrt{\mu_{n-1}}, \sqrt{\lambda_{n}}\right] \subset\left(\sqrt{\mu_{n-1}}, \sqrt{\lambda_{n}^{\prime}}\right)$. Hence, 
$\sigma_{1}(0) \tilde{v}\left(0, \xi^{2}\right) \tilde{u}_{x}\left(0, \xi^{2}\right)-\sigma_{2}(0) \tilde{u}\left(0, \xi^{2}\right) \tilde{v}_{x}\left(0, \xi^{2}\right) \neq 0$ for $\lambda \in\left(\sqrt{\mu_{n-1}}, \sqrt{\lambda_{n}}\right]$ and this implies that $G(\xi)$ is well-defined in this interval. By use of the mean value theorem on the interval $\left[\sqrt{\mu_{n-1}^{\epsilon}}, \sqrt{\lambda_{n}}\right]$, (where $\sqrt{\mu_{n-1}^{\epsilon}}=\sqrt{\mu_{n-1}}+\epsilon$ for enough small $\epsilon>0$ ), we have

$$
G\left(\sqrt{\lambda_{n}}\right)-G\left(\sqrt{\mu_{n-1}^{\epsilon}}\right)=\left.\left(\sqrt{\lambda_{n}}-\sqrt{\mu_{n-1}^{\epsilon}}\right) \frac{\partial G(\xi)}{\partial \xi}\right|_{\xi=\alpha_{n}},
$$

for some $\alpha_{n} \in\left(\sqrt{\mu_{n-1}^{\epsilon}}, \sqrt{\lambda_{n}}\right)$. From (3.27), one has $\sqrt{\mu_{n-1}^{\epsilon}} \rightarrow \sqrt{\mu_{n-1}}$ and $G\left(\sqrt{\mu_{n-1}^{\epsilon}}\right) \rightarrow 0$ as $\epsilon \rightarrow 0$. Then by using Equation (3.16) and the expression (3.23) of $\frac{\partial F(\lambda)}{\partial \lambda}$, we obtain

$$
\left(\sqrt{\lambda_{n}}-\sqrt{\mu_{n-1}}\right)=\left(\frac{\partial G}{\partial \xi}\left(\alpha_{n}\right)\right)^{-1}\left(\frac{1}{M \lambda_{n}}\right)
$$

where

$$
\left(\frac{\partial G}{\partial \xi}\left(\alpha_{n}\right)\right)^{-1}=\frac{\left(\sigma_{1}(0) \tilde{v}\left(0, \alpha_{n}^{2}\right) \tilde{u}_{x}\left(0, \alpha_{n}^{2}\right)-\sigma_{2}(0) \tilde{u}\left(0, \alpha_{n}^{2}\right) \tilde{v}_{x}\left(0, \alpha_{n}^{2}\right)\right)^{2}}{2 \alpha_{n}\left(\tilde{v}^{2}\left(0, \alpha_{n}^{2}\right) \int_{-1}^{0} \rho_{1}(s) \tilde{u}^{2}\left(s, \alpha_{n}^{2}\right) d s+\tilde{u}^{2}\left(0, \alpha_{n}^{2}\right) \int_{0}^{1} \rho_{2}(s) \tilde{v}^{2}\left(s, \alpha_{n}^{2}\right) d s\right)}
$$

Taking into account the asymptotes (3.24) and (3.25), one has

$$
\alpha_{n}\left(\sigma_{1}(0) \tilde{v}\left(0, \alpha_{n}^{2}\right) \tilde{u}_{x}\left(0, \alpha_{n}^{2}\right)-\sigma_{2}(0) \tilde{u}\left(0, \alpha_{n}^{2}\right) \tilde{v}_{x}\left(0, \alpha_{n}^{2}\right)\right)=a_{1} a_{2} \Psi\left(\alpha_{n}\right),
$$

where

$$
\Psi(\xi)=\left(\left(\frac{\rho_{1}(0) \sigma_{1}(0)}{\rho_{2}(0) \sigma_{2}(0)}\right)^{\frac{1}{4}} \sin \left(\xi \gamma_{2}\right) \cos \left(\xi \gamma_{1}\right)+\left(\frac{\rho_{1}(0) \sigma_{1}(0)}{\rho_{2}(0) \sigma_{2}(0)}\right)^{-\frac{1}{4}} \sin \left(\xi \gamma_{1}\right) \cos \left(\xi \gamma_{2}\right)\right)[1]
$$

$[1]=1+\mathcal{O}\left(\frac{1}{\xi}\right)$. Hence, by the Riemann-Lebesgue Lemma,

$$
\frac{1}{\alpha_{n}}\left(\frac{\partial G}{\partial \xi}\left(\alpha_{n}\right)\right)^{-1}=\frac{2\left(\Psi\left(\alpha_{n}\right)\right)^{2}}{\left(\gamma_{1}\left(\rho_{1}(0) \sigma_{1}(0)\right)^{-\frac{1}{2}} \sin ^{2}\left(\alpha_{n} \gamma_{1}\right)+\gamma_{2}\left(\rho_{2}(0) \sigma_{2}(0)\right)^{-\frac{1}{2}} \sin ^{2}\left(\alpha_{n} \gamma_{2}\right)\right)[1]}
$$

It is clear that if $\left|\Psi\left(\alpha_{n}\right)\right|>\tau_{1}$ for some $\tau_{1}>0$ and all $n \geq 1$, then

$$
\frac{1}{\alpha_{n}}\left(\frac{\partial G}{\partial \xi}\left(\alpha_{n}\right)\right)^{-1} \asymp 1
$$

and hence, by (3.46), we get

$$
\left(\sqrt{\lambda_{n}}-\sqrt{\mu_{n-1}}\right) \asymp \frac{\alpha_{n}}{\lambda_{n}} .
$$

Since $\sqrt{\lambda_{n-1}}<\alpha_{n}<\sqrt{\lambda_{n}}$, then from (3.31), one has

$$
\alpha_{n} \sim \frac{n \pi}{\gamma_{1}+\gamma_{2}}, \text { as } n \rightarrow \infty \text {. }
$$


Therefore, (3.43) follows from the asymptotics (3.31), (3.51) and (3.52).

Now, we assume that $\left|\Psi\left(\alpha_{n_{k}}\right)\right| \rightarrow 0$ as $k \rightarrow \infty$, for some subsequence $\left(\alpha_{n_{k}}\right)$ of $\left(\alpha_{n}\right)$. Then from (3.47), $\alpha_{n_{k}}$ tends to the square root of an eigenvalue of the regular problem (3.1) for $M=0$.

In view of Proposition 3.5, $\sqrt{\lambda_{n_{k}-1}^{\prime}}<\sqrt{\mu_{n_{k}-1}}<\alpha_{n_{k}}<\sqrt{\lambda_{n_{k}}}<\sqrt{\lambda_{n_{k}}^{\prime}}$, and hence, $\alpha_{n_{k}}$ tends to $\sqrt{\lambda_{n_{k}-1}^{\prime}}$ or $\sqrt{\lambda_{n_{k}}^{\prime}}$. According to (3.45), two cases must be examined.

Case 1 - Assume that for large $k \geq 1$ and $n_{k} \in \Omega,\left|\sqrt{\mu_{n_{k}}}-\sqrt{\mu_{n_{k}}-1}\right| \geq \tilde{\tau}_{0}$, for some $\tilde{\tau}_{0}>0$. Under this assumption, we prove (3.50). Firstly, if $\left|\alpha_{n_{k}}-\sqrt{\lambda_{n_{k}}^{\prime}}\right|$ tends to zero, then

$$
\left|\sqrt{\lambda_{n_{k}}}-\sqrt{\lambda_{n_{k}}^{\prime}}\right| \rightarrow 0 \text {, i.e., } \Psi\left(\sqrt{\lambda_{n_{k}}}\right) \rightarrow 0 \text { as } k \rightarrow \infty \text {. }
$$

On the other hand by (3.24), (3.25) and the characteristic equation (3.16), one gets $\Psi\left(\sqrt{\lambda_{n_{k}}}\right)=M\left(\rho_{1}(0) \sigma_{1}(0)\right)^{-\frac{1}{4}}\left(\rho_{2}(0) \sigma_{2}(0)\right)^{-\frac{1}{4}} \sqrt{\lambda_{n_{k}}}\left(\sin \left(\sqrt{\lambda_{n_{k}}} \gamma_{1}\right) \sin \left(\sqrt{\lambda_{n_{k}}} \gamma_{2}\right)\right)[1]$

and hence, by (3.53) $) \sin \left(\sqrt{\lambda_{n_{k}}} \gamma_{1}\right)[1] \rightarrow 0$ or $\sin \left(\sqrt{\lambda_{n_{k}}} \gamma_{2}\right)[1] \rightarrow 0$, as $k \rightarrow \infty$. Again by (3.53) together with (3.48), we have

$$
\left(\left|\sin \left(\sqrt{\lambda_{n_{k}}} \gamma_{1}\right)\right|+\left|\sin \left(\sqrt{\lambda_{n_{k}}} \gamma_{2}\right)\right|\right)[1] \rightarrow 0, \text { as } k \rightarrow \infty
$$

This means that $\sqrt{\lambda_{n_{k}}}$ tends simultaneously to the square root of eigenvalues of the two Problems (3.8) and (3.9). Since $\left|\sqrt{\mu_{n_{k}}}-\sqrt{\mu_{n_{k}-1}}\right| \geq \tilde{\tau}_{0}$, then in view of assertion (i) of Lemma 3.6, we have $\left|\sqrt{\lambda_{n_{k}}}-\sqrt{\mu_{n_{k}-1}}\right|$ tends to zero and $\sqrt{\mu_{n_{k}-2}}$ close to $\sqrt{\mu_{n_{k}-1}}$ (or $\left|\sqrt{\lambda_{n_{k}}}-\sqrt{\mu_{n_{k}}}\right| \rightarrow 0$ and $\left|\sqrt{\mu_{n_{k}+1}}-\sqrt{\mu_{n_{k}}}\right| \rightarrow 0$ ). According to Proposition 3.5, $\sqrt{\lambda_{n_{k}-1}^{\prime}} \in\left(\sqrt{\mu_{n_{k}-2}}, \sqrt{\mu_{n_{k}-1}}\right)$, thus by $(3.53)$, one has

$$
\left|\sqrt{\lambda_{n_{k}}^{\prime}}-\sqrt{\lambda_{n_{k}-1}^{\prime}}\right| \rightarrow 0, \text { as } k \rightarrow \infty
$$

This is in contradiction with the asymptotic estimate (3.32).

Now, if $\left|\alpha_{n_{k}}-\sqrt{\lambda_{n_{k}-1}^{\prime}}\right|$ converge to zero, then $\left|\alpha_{n_{k}}-\sqrt{\mu_{n_{k}-1}}\right|$ tends to zero, and hence, $\sin \left(\alpha_{n_{k}} \gamma_{1}\right)[1] \rightarrow 0$ or $\sin \left(\alpha_{n_{k}} \gamma_{2}\right)$ [1] $\rightarrow 0$ as $k \rightarrow \infty$. As above, since $\Psi\left(\alpha_{n_{k}}\right) \rightarrow 0$ as $k \rightarrow \infty$, then by (3.48),

$$
\left(\left|\sin \left(\alpha_{n_{k}} \gamma_{1}\right)\right|+\left|\sin \left(\alpha_{n_{k}} \gamma_{2}\right)\right|\right)[1] \rightarrow 0, \text { as } k \rightarrow \infty \text {. }
$$

As before in view of the assumption $\left|\sqrt{\mu_{n_{k}}}-\sqrt{\mu_{n_{k}-1}}\right|>\tilde{\tau}_{0}$, (3.54) implies

$$
\left|\alpha_{n_{k}}-\sqrt{\mu_{n_{k}-1}}\right| \rightarrow 0 \text { and }\left|\sqrt{\mu_{n_{k}-1}}-\sqrt{\mu_{n_{k}-2}}\right| \rightarrow 0 \text {, as } k \rightarrow \infty \text {. }
$$

Let us recall from the expression (3.15) of $F(\lambda)$ that

$$
F\left(\xi^{2}\right)=F_{1}\left(\xi^{2}\right)-F_{2}\left(\xi^{2}\right)
$$


where $F_{1}$ and $F_{2}$ are defined by (3.29). This means that $\left(\frac{\partial G}{\partial \xi}(\xi)\right)^{-1}$ can be expressed in the form

$$
\left(\frac{\partial G}{\partial \xi}(\xi)\right)^{-1}=\frac{-\left(F_{1}\left(\xi^{2}\right)-F_{2}\left(\xi^{2}\right)\right)^{2}}{2 \xi\left(\frac{\partial F_{1}}{\partial \xi}\left(\xi^{2}\right)-\frac{\partial F_{2}}{\partial \xi}\left(\xi^{2}\right)\right)}, \text { for } \xi \in\left(\sqrt{\mu_{n_{k}-1}}, \sqrt{\lambda_{n_{k}}}\right]
$$

Similarly to the proof of Lemma 3.4, we can prove that $F_{1}\left(\xi^{2}\right)$ is a decreasing function from $+\infty$ to $-\infty$ in all the intervals $\left(-\infty, \sqrt{\mu_{n}^{-}}\right)$and $\left(\sqrt{\mu_{n}^{-}}, \sqrt{\mu_{n+1}^{-}}\right)$, while, $F_{2}\left(\xi^{2}\right)$ is an increasing function from $-\infty$ to $+\infty$ along each of the intervals $\left(-\infty, \sqrt{\mu_{n}^{+}}\right)$and $\left(\sqrt{\mu_{n}^{+}}, \sqrt{\mu_{n+1}^{+}}\right), n \geq 1$. By $(\underline{3.55}), \sqrt{\mu_{n_{k}-2}}$ close to $\sqrt{\mu_{n_{k}-1}}$, then in view of assertion (i) of Lemma 3.6, we may assume that $\mu_{n_{k}-2}$ and $\mu_{n_{k}-1}$ are eigenvalues of Problems (3.8) and (3.9), respectively (the other case can be handled in a same way), i.e.,

$$
\lim _{\xi \rightarrow \sqrt{\mu_{n_{k}-2}}+0} F_{1}\left(\xi^{2}\right)=+\infty, \quad \lim _{\xi \rightarrow \sqrt{\mu_{n_{k}-1}}+0} F_{2}\left(\xi^{2}\right)=-\infty .
$$

Therefore, by (3.55) and (3.57), for each $\alpha_{n_{k}}$ in a sufficiently small right neighborhood of $\sqrt{\mu_{n_{k}-1}}$, one gets

$$
F_{1}\left(\alpha_{n_{k}}^{2}\right) F_{2}\left(\alpha_{n_{k}}^{2}\right)<0 .
$$

It is clear that the above together with (3.56) and (3.58) imply that

$$
\left(\frac{\partial G}{\partial \xi}\left(\alpha_{n_{k}}\right)\right)^{-1} \asymp \frac{\left(F_{1}\left(\alpha_{n_{k}}^{2}\right)\right)^{2}+\left(F_{2}\left(\alpha_{n_{k}}^{2}\right)\right)^{2}}{\alpha_{n_{k}}\left(\frac{\partial F_{2}}{\partial \xi}\left(\alpha_{n_{k}}^{2}\right)-\frac{\partial F_{1}}{\partial \xi}\left(\alpha_{n_{k}}^{2}\right)\right)}
$$

Thus, combining (3.49) with (3.59), we obtain

$$
\frac{1}{\alpha_{n_{k}}}\left(\frac{\partial G}{\partial \xi}\left(\alpha_{n_{k}}\right)\right)^{-1} \asymp \frac{\left(\sin ^{2}\left(\alpha_{n_{k}} \gamma_{2}\right) \cos ^{2}\left(\alpha_{n_{k}} \gamma_{1}\right)+\sin ^{2}\left(\alpha_{n_{k}} \gamma_{1}\right) \cos ^{2}\left(\alpha_{n_{k}} \gamma_{2}\right)\right)[1]}{\left(\sin ^{2}\left(\alpha_{n_{k}} \gamma_{1}\right)+\sin ^{2}\left(\alpha_{n_{k}} \gamma_{2}\right)\right)[1]}
$$

and hence, by (3.54), the desired estimate (3.50) follows.

Case 2- Now, suppose that $\sqrt{\mu_{n_{k}-1}}$ close to $\sqrt{\mu_{n_{k}}}$. This implies that for $\alpha_{n_{k}} \in\left(\sqrt{\mu_{n_{k}-1}}, \sqrt{\mu_{n_{k}}}\right)$,

$$
\left(\left|\sin \left(\alpha_{n_{k}} \gamma_{1}\right)\right|+\left|\sin \left(\alpha_{n_{k}} \gamma_{2}\right)\right|\right)[1] \rightarrow 0, \text { as } k \rightarrow \infty \text {. }
$$

In this case, we prove for sufficiently large $k$, that

$$
\frac{1}{\alpha_{n_{k}}}\left(\frac{\partial G}{\partial \xi}\left(\alpha_{n_{k}}\right)\right)^{-1} \leq C, \quad C>0
$$

Obviously, under the above assumption, we have $\left|\sqrt{\mu_{n_{k}-2}}-\sqrt{\mu_{n_{k}-1}}\right|>\tilde{\tau}_{0}, \tilde{\tau}_{0}>0$. 
As above, we may assume that $\mu_{n_{k}-1}$ and $\mu_{n_{k}}$ are eigenvalues of Problems (3.8) and (3.9), respectively. Hence, analogously to the first case we have the two limits,

$$
\lim _{\xi \rightarrow \sqrt{\mu_{n_{k}}-1}+0} F_{1}\left(\xi^{2}\right)=+\infty, \quad \lim _{\xi \rightarrow \sqrt{\mu_{n_{k}}}-0} F_{2}\left(\xi^{2}\right)=+\infty
$$

where $F_{1}$ and $F_{2}$ are defined by (3.29). Therefore, by (3.61), for sufficiently large $k$ and each $\alpha_{n_{k}} \in\left(\sqrt{\mu_{n_{k}-1}}, \sqrt{\mu_{n_{k}}}\right)$,

$$
F_{1}\left(\alpha_{n_{k}}^{2}\right) F_{2}\left(\alpha_{n_{k}}^{2}\right)>0 .
$$

Thus by (3.56), one has

$$
\left(\frac{\partial G}{\partial \xi}\left(\alpha_{n_{k}}\right)\right)^{-1} \leq \frac{\left(F_{1}\left(\alpha_{n_{k}}^{2}\right)\right)^{2}+\left(F_{2}\left(\alpha_{n_{k}}^{2}\right)\right)^{2}}{2 \alpha_{n_{k}}\left(\frac{\partial F_{2}}{\partial \xi}\left(\alpha_{n_{k}}^{2}\right)-\frac{\partial F_{1}}{\partial \xi}\left(\alpha_{n_{k}}^{2}\right)\right)}
$$

In a similar way as the first one, by use of (3.49) and (3.62), the desired estimate (3.60) follows.

Therefore, combining (3.31), (3.46), (3.52) with (3.60), we get for sufficiently large $k$,

$$
\left(\sqrt{\lambda_{n_{k}}}-\sqrt{\mu_{n_{k}-1}}\right) \leq \frac{C}{n_{k}}, \quad C>0 .
$$

As mentioned above, $\mu_{n_{k}-1}$ and $\mu_{n_{k}}$ are eigenvalues of Problems (3.8) and (3.9), respectively. Then by (3.10) and (3.24),

$$
\sqrt{\lambda_{n_{k}}}\left|\tilde{u}\left(0, \lambda_{n_{k}}\right)\right| \sim a_{1}\left(\rho_{1}(0) \sigma_{1}(0)\right)^{-\frac{1}{4}} \gamma_{1} \delta_{n_{k}}^{-}, \text {as } k \rightarrow \infty,
$$

where $\delta_{n_{k}}^{-}=\sqrt{\lambda_{n_{k}}}-\sqrt{\mu_{n_{k}-1}}$. Hence, if $\delta_{n_{k}}^{-}<\frac{C}{n_{k}}$, then by Lemma 3.6, $\left|\tilde{u}\left(0, \lambda_{n_{k}}\right)\right| \sim\left|\tilde{v}\left(0, \lambda_{n_{k}}\right)\right|$, i.e.,

$$
\delta_{n_{k}}^{-} \sim \delta_{n_{k}}^{+}, \text {as } k \rightarrow \infty
$$

where $\delta_{n_{k}}^{+}=\sqrt{\mu_{n_{k}}}-\sqrt{\lambda_{n_{k}}}$. This implies that $\left|\sqrt{\mu_{n_{k}}}-\sqrt{\mu_{n_{k}-1}}\right|<\frac{C}{n_{k}}, C>0$, and this is in contradiction with (3.45). Thus by (3.63),

$$
\sqrt{\lambda_{n_{k}}}-\sqrt{\mu_{n_{k}-1}}=\mathcal{O}\left(\frac{1}{n_{k}}\right)
$$

which proves (3.43).

Now we prove the estimate (3.6). If $\frac{\gamma_{1}}{\gamma_{2}} \notin \mathbb{Q}$, then for every $\varepsilon>0$ and $A>0$, there exist integers $j>A, k>A$ such that

$$
\left|\frac{j \gamma_{1}}{\gamma_{2}}-k\right| \leq \varepsilon
$$


If $\frac{\gamma_{1}}{\gamma_{2}} \in \mathbb{Q}$, then $\frac{\gamma_{1}}{\gamma_{2}}=\frac{j}{k}$, for some integers $j, k \geq 1$. Hence, there exist two subsequences of eigenvalues $\left(\sqrt{\mu_{\varphi(j)}^{-}}\right)$and $\left(\sqrt{\mu_{\varphi(k)}^{+}}\right)$(where $\sqrt{\mu_{n}^{-}}$and $\sqrt{\mu_{n}^{+}}$satisfy $(\underline{3.10})$ ) such that

$$
\left|\sqrt{\mu_{\varphi(k)}^{+}}-\sqrt{\mu_{\varphi(j)}^{-}}\right| \rightarrow 0, \text { as } j, k \rightarrow \infty
$$

or equivalently,

$$
\sqrt{\mu_{\varphi(n)}}-\sqrt{\mu_{\varphi(n)-1}} \rightarrow 0, \text { as } n \rightarrow \infty .
$$

According to Proposition 3.5, there exists a subsequence $\left(\lambda_{\varphi(n)}\right)$ such that $\lambda_{\varphi(n)} \in\left[\mu_{\varphi(n)-1}, \mu_{\varphi(n)}\right]$. Then

$$
\sqrt{\mu_{\varphi(n)}}-\sqrt{\lambda_{\varphi(n)}} \rightarrow 0, \text { as } n \rightarrow \infty
$$

It is clear from (3.64) and Lemma 3.6, that $(\varphi(n)+1) \in \Omega$, where $\Omega$ is defined by (3.44). Hence, by (3.43),

$$
\sqrt{\lambda_{\varphi(n)+1}}-\sqrt{\mu_{\varphi(n)}}=\mathcal{O}\left(\frac{1}{n}\right) .
$$

We shall prove for some $C>0$, that

$$
\sqrt{\mu_{\varphi(n)}}-\sqrt{\lambda_{\varphi(n)}} \leq \frac{C}{n}, \text { as } n \rightarrow \infty
$$

First, we suppose that $\varphi(n) \in \Omega$, then

$$
\sqrt{\lambda_{\varphi(n)}}-\sqrt{\mu_{\varphi(n)-1}}=\mathcal{O}\left(\frac{1}{n}\right)
$$

As before, without loss of generality, we may assume that $\mu_{\varphi(n)-1}$ and $\mu_{\varphi(n)}$ are eigenvalues of Problems (3.8) and (3.9), respectively. This, (3.10) and (3.68), yields

$$
\left|\sin \left(\sqrt{\lambda_{\varphi(n)}} \gamma_{1}\right)\right| \sim \frac{C}{n}, \text { for some } C>0 \text { and large } n \in \mathbb{N}^{*}
$$

According to assertion (ii) of Lemma 3.6 and (3.69), we have

$$
\left|\sin \left(\sqrt{\lambda_{\varphi(n)}} \gamma_{2}\right)\right| \sim \frac{C^{\prime}}{n}, \text { for some } C^{\prime}>0
$$

Since $\mu_{\varphi(n)}$ is an eigenvalue of Problem (3.9), then by (3.10), (3.65) and (3.70),

$$
\sqrt{\mu_{\varphi(n)}}-\sqrt{\lambda_{\varphi(n)}}=\mathcal{O}\left(\frac{1}{n}\right) .
$$

Now, if $\varphi(n) \notin \Omega$, clearly from (3.10) and (3.44), we have $\sqrt{\mu_{\varphi(n)}}-\sqrt{\mu_{\varphi(n)-1}}<\frac{C}{n}$ for some $C>0$ and large $n \in \mathbb{N}^{*}$. Using this and (3.30), one gets

$$
\sqrt{\mu_{\varphi(n)}}-\sqrt{\lambda_{\varphi(n)}}<\frac{C}{n} \text { and } \sqrt{\lambda_{\varphi(n)}}-\sqrt{\mu_{\varphi(n)-1}}<\frac{C}{n} .
$$


Hence, from this and (3.71), the estimate (3.67) holds. Combining (3.66) and (3.67), the desired estimate (3.6) follows.

It is easily seen from (3.30) and assertion $(i)$ of Lemma 3.6, that the estimate (3.7) holds. This ends up the proof of the theorem.

Proceeding as above, we have the following result:

Corollary 3.7 Let $\left(\mu_{n_{k}}\right)$ be a subsequence of $\left(\mu_{n}\right)$.

If $\sqrt{\mu_{n_{k}}}-\sqrt{\mu_{n_{k}-1}} \rightarrow 0$ as $k \rightarrow \infty$, then $\sqrt{\lambda_{n_{k}+1}}-\sqrt{\lambda_{n_{k}}}=\mathcal{O}\left(\frac{1}{k}\right), \sqrt{\mu_{n_{k}}}-\sqrt{\lambda_{n_{k}}} \leq \frac{C}{k}$ and $\sqrt{\lambda_{n_{k}}}-\sqrt{\mu_{n_{k}-1}} \leq \frac{C}{k}$ for some $C>0$.

Moreover, if $\left|\sqrt{\mu_{n_{k}}}-\sqrt{\mu_{n_{k}-1}}\right|>C$ for some $C>0$ and large $k$, then $\left|\sqrt{\lambda_{n_{k}+1}}-\sqrt{\lambda_{n_{k}}}\right|>C^{\prime}$ for all $k$.

We establish now the asymptotic behavior of the eigenfunctions $\left(\phi_{n}(x)\right)_{n \geq 1}$ of the eigenvalue problem (3.1).

Proposition 3.8 Define the set

$$
\Lambda=\left\{n \in \mathbb{N}^{*} \text { such that } \mu_{n} \in \Gamma^{*}\right\},
$$

where the set $\Gamma^{*}$ is defined by (3.12). Then the associated eigenfunctions $\left(\phi_{n}(x)\right)_{n \geq 1}$ of the eigenvalue problem (3.1) satisfy the following asymptotic estimates:

i) For $n \in \Lambda$,

$$
\phi_{n}(x)=\left\{\begin{array}{l}
-a_{1} a_{2}\left(\frac{\rho_{1}(x) \sigma_{1}(x)}{\rho_{2}(0) \sigma_{2}(0)}\right)^{-\frac{1}{4}} \frac{\cos \left(\sqrt{\lambda_{n}} \gamma_{2}\right) \sin \left(\sqrt{\lambda_{n}} \int_{-1}^{x} \sqrt{\frac{\rho_{1}(t)}{\sigma_{1}(t)}} d t\right)}{\sqrt{\lambda_{n}}}[1], \quad x \in[-1,0], \\
a_{1} a_{2}\left(\frac{\rho_{2}(x) \sigma_{2}(x)}{\rho_{1}(0) \sigma_{1}(0)}\right)^{-\frac{1}{4}} \frac{\cos \left(\sqrt{\lambda_{n}} \gamma_{1}\right) \sin \left(\sqrt{\lambda_{n}} \int_{x}^{1} \sqrt{\frac{\rho_{2}(t)}{\sigma_{2}(t)}} d t\right)}{\sqrt{\lambda_{n}}}[1], \quad x \in[-1,0],
\end{array}\right.
$$

where $[1]=1+\mathcal{O}\left(\frac{1}{n}\right), \gamma_{1}=\int_{-1}^{0} \sqrt{\frac{\rho_{1}(x)}{\sigma_{1}(x)}} d x, \gamma_{2}=\int_{0}^{1} \sqrt{\frac{\rho_{2}(x)}{\sigma_{2}(x)}} d x$ and the constants $a_{i} \quad(i=1,2)$ are defined by (3.26).

ii) For $n \in \mathbb{N}^{*} \backslash \Lambda$,

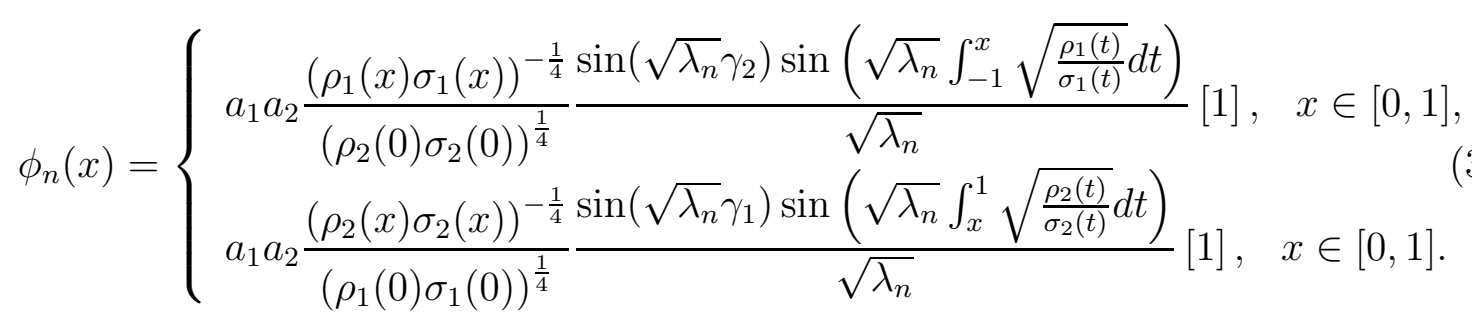


Proof. It is clear that for all $n \in \Lambda, \tilde{u}\left(0, \lambda_{n}\right)=\tilde{v}\left(0, \lambda_{n}\right)=0$. Then, from (3.5) together with the last condition in (3.1), the corresponding eigenfunctions $\left(\phi_{n}(x)\right)_{n \in \Lambda}$ can be written in the form

$$
\phi_{n}(x)= \begin{cases}\sigma_{2}(0) \tilde{v}_{x}\left(0, \lambda_{n}\right) \tilde{u}\left(x, \lambda_{n}\right), & -1 \leq x \leq 0 \\ \sigma_{1}(0) \tilde{u}_{x}\left(0, \lambda_{n}\right) \tilde{v}\left(x, \lambda_{n}\right), & 0 \leq x \leq 1\end{cases}
$$

Therefore, the asymptotes (3.73) are simple deductions from the asymptotes (3.24), (3.25) and (3.75). Now, if $n \in \mathbb{N}^{*} \backslash \Lambda$, we have $\tilde{u}\left(0, \lambda_{n}\right) \neq 0$ and $\tilde{v}\left(0, \lambda_{n}\right) \neq 0$, since otherwise $\lambda_{n}$ is not an eigenvalue of Problem (3.1) (see the second case in the proof of Lemma 3.1). We set

$$
\phi_{n}(x)=\sqrt{\lambda_{n}} \widetilde{U}\left(x, \lambda_{n}\right),
$$

where $\widetilde{U}\left(x, \lambda_{n}\right)$ is defined by (3.14). From the asymptotes (3.24), (3.25) and (3.76), a straightforward computation gives the asymptotes (3.74).

\section{Riesz Basis And Asymmetric Space}

In this section we give results concerning the Riesz basis and the asymmetric space that we will need later to prove the observability inequality. Let us begin with the following result due to C. Baiocchi et al. [5]:

Theorem 4.1 Let $\left(\omega_{n}\right)$ be a strictly increasing sequence satisfying for some $\delta>0$ the condition:

$$
\omega_{n+2}-\omega_{n}>2 \delta, \text { for all } n .
$$

Fix a number $0<\delta^{\prime} \leq \delta$, and set

$$
\begin{aligned}
& A=\left\{n \in \mathbb{Z}, \quad \omega_{n}-\omega_{n-1} \geq \delta^{\prime} \text { and } \omega_{n+1}-\omega_{n}<\delta^{\prime}\right\}, \\
& B=\left\{n \in \mathbb{Z}, \quad \omega_{n}-\omega_{n-1} \geq \delta^{\prime} \text { and } \omega_{n+1}-\omega_{n} \geq \delta^{\prime}\right\} .
\end{aligned}
$$

Then the following estimates hold for every bounded interval I of length $|I|>2 \pi D^{+}$: there exist two constants $C_{1}>0$ and $C_{2}>0$ such that for any $f=\sum_{n \in \mathbb{Z}} a_{n} e^{i \omega_{n} t}$,

$$
\begin{aligned}
& C_{1}\left(\sum_{n \in A}\left[\left(\omega_{n+1}-\omega_{n}\right)^{2}\left(\left|a_{n}\right|^{2}+\left|a_{n+1}\right|^{2}\right)+\left|a_{n+1}+a_{n}\right|^{2}\right]+\sum_{n \in B}\left|a_{n}\right|^{2}\right) \leq \int_{I}|f(t)|^{2} d t \\
& \leq C_{2}\left(\sum_{n \in A}\left[\left(\omega_{n+1}-\omega_{n}\right)^{2}\left(\left|a_{n}\right|^{2}+\left|a_{n+1}\right|^{2}\right)+\left|a_{n+1}+a_{n}\right|^{2}\right]+\sum_{n \in B}\left|a_{n}\right|^{2}\right),
\end{aligned}
$$

where $D^{+}:=\lim _{r \rightarrow+\infty} \frac{n^{+}(r)}{r}$ and $n^{+}(r)$ denotes the largest number of terms of the sequence $\left(\omega_{n}\right)$ contained in an interval of length $r$. 
Set

$$
\sqrt{\lambda_{-n}}=-\sqrt{\lambda_{n}}, \sqrt{\mu_{-n}}=-\sqrt{\mu_{n}} \text { and } \Phi_{-n}=\Phi_{n} \text {, for all } n \in \mathbb{N}^{*} \text {. }
$$

In light of Theorem 3.2 and Corollary 3.7, let $\sigma^{*}$ be the set of elements of all subsequences $\left(\lambda_{n_{k}}\right)$ which satisfy $\sqrt{\lambda_{n_{k}+1}}-\sqrt{\lambda_{n_{k}}}=\mathcal{O}\left(\frac{1}{k}\right)$, i.e.,

$$
\sigma^{*}=\left\{\lambda_{n_{k}} \text { such that } \sqrt{\lambda_{n_{k}+1}}-\sqrt{\lambda_{n_{k}}}=\mathcal{O}\left(\frac{1}{k}\right)\right\} \text {. }
$$

Denote by

$$
A=\left\{n \in \mathbb{Z}^{*} \text { such that } \lambda_{n} \in \sigma^{*}\right\}
$$

and

$$
B=\left\{n \in \mathbb{Z}^{*},(n-1) \notin A \text { and } n \notin A\right\} .
$$

Note that if $n \in A$, then $(n+1) \notin A$ and $(n+1) \notin B$. Thus

$$
\mathbb{Z}^{*}=A \cup B \cup\{n+1, \quad n \in A\} .
$$

Moreover, it is easily seen from Corollary 3.7, that for large $n \in A,\left|\sqrt{\mu_{n}}-\sqrt{\mu_{n-1}}\right|$ tends to zero.

Lemma 4.2 For $T>2\left(\gamma_{1}+\gamma_{2}\right)$, where $\gamma_{1}=\int_{-1}^{0} \sqrt{\frac{\rho_{1}(x)}{\sigma_{1}(x)}} d x$ and $\gamma_{2}=\int_{0}^{1} \sqrt{\frac{\rho_{2}(x)}{\sigma_{2}(x)}} d x$, there exist two positive constants $C_{1}$ and $C_{2}$ such that for $f=\sum_{n \in \mathbb{Z}^{*}} a_{n} e^{i \sqrt{\lambda_{n}} t}$, we have

$$
\begin{aligned}
& C_{1}\left(\sum_{n \in A}\left[\left(\left|a_{n}\right|^{2}+\left|a_{n+1}\right|^{2}\right) \delta_{n}^{2}+\left|a_{n+1}+a_{n}\right|^{2}\right]+\sum_{n \in B}\left|a_{n}\right|^{2}\right) \leq \int_{0}^{T}\left|\sum_{n \in \mathbb{Z}^{*}} a_{n} e^{i \sqrt{\lambda_{n}} t}\right|^{2} d t \\
& \leq C_{2}\left(\sum_{n \in A}\left[\left(\left|a_{n}\right|^{2}+\left|a_{n+1}\right|^{2}\right) \delta_{n}^{2}+\left|a_{n+1}+a_{n}\right|^{2}\right]+\sum_{n \in B}\left|a_{n}\right|^{2}\right) .
\end{aligned}
$$

Proof. By (3.7), we have for $n \in A, \sqrt{\lambda_{n}}-\sqrt{\lambda_{n-1}} \geq \delta^{\prime}$ for some $\delta^{\prime}<\delta$. Hence the second condition in the set $A$ given in Theorem 4.1 holds for $\omega_{n}=\sqrt{\lambda_{n}}$.

In view of Proposition 3.5, $\frac{n^{+}\left(\sqrt{\lambda_{n-1}^{\prime}}\right)}{\sqrt{\lambda_{n}^{\prime}}} \leq \frac{n^{+}\left(\sqrt{\lambda_{n}}\right)}{\sqrt{\lambda_{n}}} \leq \frac{n^{+}\left(\sqrt{\lambda_{n}^{\prime}}\right)}{\sqrt{\lambda_{n-1}^{\prime}}}$, where $\lambda_{n}^{\prime}(n \geq 1)$ denote the eigenvalues of the regular problem (3.1) for $M=0$, and $n^{+}$is defined in Theorem 4.1. Using the asymptote (3.32), we find $D^{+}=\frac{\gamma_{1}+\gamma_{2}}{\pi}$. By setting $\omega_{n}=\sqrt{\lambda_{n}}$,

$\delta_{n}=\sqrt{\lambda_{n+1}}-\sqrt{\lambda_{n}}, n \in \mathbb{Z}^{*}$, in view of (3.6) and (3.7), we are in the conditions of Theorem 4.1, and hence, Inequality (4.5) follows.

Define the scale of Hilbert spaces $\left(X_{\alpha},\|\cdot\|_{\alpha}\right), \alpha \in \mathbb{R}$ :

$$
X_{\alpha}=\left\{Y: Y=\sum_{n \in \mathbb{N}^{*}} a_{n} \Phi_{n} \text { with }\|Y\|_{\alpha}^{2}=\sum_{n \in \mathbb{N}^{*}}\left|a_{n}\right|^{2} \lambda_{n}^{2 \alpha}\left\langle\Phi_{n}, \Phi_{n}\right\rangle_{\mathcal{W}}<\infty\right\}
$$

endowed with the norm $\|\cdot\|_{\alpha}$, where $\mathcal{W}$ is defined by (2.1) and $\Phi_{n}$ are the eigenfunctions of the operator $\mathcal{A}$. 


\section{Proposition 4.3 We have}

$$
\begin{gathered}
\left\langle\Phi_{n}, \Phi_{n}\right\rangle_{\mathcal{W}}=\mathcal{O}(1), n \in \Lambda \cup\left(B \cap \mathbb{N}^{*}\right), \\
\left\langle\Phi_{n}, \Phi_{n}\right\rangle_{\mathcal{W}} \leq \frac{C}{n^{2}}, \text { for large } n \in\left(A \cap \mathbb{N}^{*}\right) \backslash \Lambda \text { and } C>0,
\end{gathered}
$$

and

$$
\left\langle\Phi_{n+1}, \Phi_{n+1}\right\rangle_{\mathcal{W}}=\mathcal{O}\left(\frac{1}{n^{2}}\right) \text {, for large } n \in A \cap \mathbb{N}^{*} .
$$

where the sets $\Lambda, A$ and $B$ are defined by (3.72), (4.2) and (4.3), respectively.

Proof. In view of (2.3), (3.24) and (3.25), we have for $n \in \mathbb{N}^{*}$

$$
\left\|\tilde{u}\left(x, \lambda_{n}\right)\right\|_{\mathcal{V}_{1}}^{2}=\mathcal{O}(1) \text { and }\left\|\tilde{v}\left(x, \lambda_{n}\right)\right\|_{\mathcal{V}_{2}}^{2}=\mathcal{O}(1) .
$$

It is clear from (2.2) and (3.75) , that for $n \in \Lambda$

$$
\left\langle\Phi_{n}, \Phi_{n}\right\rangle_{\mathcal{W}}=\left(\sigma_{2}(0) \tilde{v}_{x}\left(0, \lambda_{n}\right)\right)^{2}\left\|\tilde{u}\left(x, \lambda_{n}\right)\right\|_{\mathcal{V}_{1}}^{2}+\left(\sigma_{1}(0) \tilde{u}_{x}\left(0, \lambda_{n}\right)\right)^{2}\left\|\tilde{v}\left(x, \lambda_{n}\right)\right\|_{\mathcal{V}_{2}}^{2} .
$$

Note that for $n \in \Lambda, \lambda_{n}=\mu_{n}$, i.e., $\tilde{u}\left(0, \lambda_{n}\right)=\tilde{v}\left(0, \lambda_{n}\right)=0$. In this case $\lambda_{n}$ is an eigenvalue of both Problems (3.8) and (3.9). This means that $\tilde{u}_{x}\left(0, \lambda_{n}\right) \neq 0$ and $\tilde{v}_{x}\left(0, \lambda_{n}\right) \neq 0$ for all $n \in \Lambda$, and hence, by (3.10), (3.24) and (3.25), we get for large $n \in \Lambda$

$$
\left|\sigma_{1}(0) \tilde{u}_{x}\left(0, \lambda_{n}\right)\right| \sim \frac{\left(\rho_{1}(0) \sigma_{1}(0)\right)^{\frac{1}{4}}}{\left(\rho_{1}(-1)\right)^{\frac{1}{4}}\left(\sigma_{1}(-1)\right)^{-\frac{3}{4}}} \text { and }\left|\sigma_{2}(0) \tilde{v}_{x}\left(0, \lambda_{n}\right)\right| \sim \frac{\left(\rho_{2}(0) \sigma_{2}(0)\right)^{\frac{1}{4}}}{\left(\rho_{2}(1)\right)^{\frac{1}{4}}\left(\sigma_{2}(1)\right)^{-\frac{3}{4}}} .
$$

From this, (4.10) and (4.11), the estimate (4.7) holds for $n \in \Lambda$.

Now for $n \in \mathbb{N}^{*} \backslash \Lambda$, using (2.2) and (3.76) , we obtain

$$
\left\langle\Phi_{n}, \Phi_{n}\right\rangle_{\mathcal{W}}=\left(\sqrt{\lambda_{n}} \tilde{v}\left(0, \lambda_{n}\right)\right)^{2}\left\|\tilde{u}\left(x, \lambda_{n}\right)\right\|_{\mathcal{V}_{1}}^{2}+\left(\sqrt{\lambda_{n}} \tilde{u}\left(0, \lambda_{n}\right)\right)^{2}\left\|\tilde{v}\left(x, \lambda_{n}\right)\right\|_{\mathcal{V}_{2}}^{2} .
$$

By Corollary 3.7, we have for large $n \in\left(A \cap \mathbb{N}^{*}\right) \backslash \Lambda$,

$$
\left|\sqrt{\lambda_{n}}-\sqrt{\mu_{n}}\right| \leq \frac{C}{n} \text { and }\left|\sqrt{\lambda_{n}}-\sqrt{\mu_{n-1}}\right| \leq \frac{C}{n} \text {, for some } C>0 .
$$

Then by use of (3.10), (3.24) and (3.25) together with (4.14), we obtain

$$
\left|\sqrt{\lambda_{n}} \tilde{u}\left(0, \lambda_{n}\right)\right| \leq \frac{C^{\prime}}{n} \text { and }\left|\sqrt{\lambda_{n}} \tilde{v}\left(0, \lambda_{n}\right)\right| \leq \frac{C^{\prime \prime}}{n} \text {, for some } C^{\prime}, C^{\prime \prime}>0 \text {. }
$$

Since for large $n \in\left(A \cap \mathbb{N}^{*}\right),\left|\sqrt{\mu_{n}}-\sqrt{\mu_{n-1}}\right|$ tends to zero, then in view of Corollary 3.7 together with (3.43),

$$
\sqrt{\lambda_{n+1}}-\sqrt{\mu_{n}}=\mathcal{O}\left(\frac{1}{n}\right) \text { and } \sqrt{\lambda_{n+1}}-\sqrt{\mu_{n-1}}=\mathcal{O}\left(\frac{1}{n}\right) \text {, for large } n \in\left(A \cap \mathbb{N}^{*}\right) .
$$


Substituting these asymptotes into (3.24) and (3.25), one gets

$$
\sqrt{\lambda_{n+1}} \tilde{u}\left(0, \lambda_{n+1}\right)=\mathcal{O}\left(\frac{1}{n}\right) \text { and } \sqrt{\lambda_{n+1}} \tilde{v}\left(0, \lambda_{n+1}\right)=\mathcal{O}\left(\frac{1}{n}\right) .
$$

It is easily seen that $\left|\sqrt{\mu_{n}}-\sqrt{\mu_{n-1}}\right|>\delta^{\prime}$ for all $n \in B$. Then again by (3.10) and (3.24) together with (3.25) and (3.43),

$$
\left|\sqrt{\lambda_{n}} \tilde{u}\left(0, \lambda_{n}\right)\right|+\left|\sqrt{\lambda_{n}} \tilde{v}\left(0, \lambda_{n}\right)\right|>C \text {, for some } C>0 \text { and large } n \in B \text {. }
$$

Therefore, from the above and (4.13), the desired estimates (4.7), (4.8) and (4.9) hold. The proposition is proved.

As a consequence of Proposition 4.3, we give other descriptions of some of the spaces $X_{\alpha}$.

Proposition 4.4 We have the following characterizations of the spaces $X_{\alpha}$ :

1. $X_{0}=D\left(\mathcal{A}^{\frac{1}{2}}\right)$ coincides topologically with the subspace $\mathcal{W}$, where $\mathcal{W}$ is defined by (2.1).

2. $X_{-\frac{1}{2}}=D\left(\mathcal{A}^{0}\right)$ coincides algebraically and topologically with the space $\mathcal{H}_{0}$, where $\mathcal{H}_{0}$ is defined by (1.5).

3. $X_{-1}=D\left(\mathcal{A}^{-\frac{1}{2}}\right)$ coincides with the dual space of $X_{0}$, it is the subspace $\mathcal{H}_{-1}$, where $\mathcal{H}_{-1}$ is defined by (1.7).

Proof. The proof is analogous to that of [8].

Let us now recall briefly how solutions of (1.1)-(1.2)-(1.3) can be developed in Fourier series. Given initial data $\left(u^{0}, v^{0}, z^{0}\right) \in X_{-\frac{1}{2}},\left(u^{1}, v^{1}, z^{1}\right) \in X_{-1}$, we compute its Fourier coefficients

$$
\tilde{e}_{n}=\frac{\left\langle\left(u^{0}, v^{0}, z^{0}\right), \Phi_{n}\right\rangle_{X_{-\frac{1}{2}}}}{\left\langle\Phi_{n}, \Phi_{n}\right\rangle_{X_{-\frac{1}{2}}}}, \quad \tilde{f}_{n}=\frac{\left\langle\left(u^{1}, v^{1}, z^{1}\right), \Phi_{n}\right\rangle_{X_{-1}}}{\left\langle\Phi_{n}, \Phi_{n}\right\rangle_{X_{-\frac{1}{2}}}},
$$

where $\Phi_{n}=\left(\phi_{n}, \phi_{n}(0)\right)$ and $\left\langle\Phi_{n}, \Phi_{n}\right\rangle_{X_{-\frac{1}{2}}}=\frac{\left\langle\Phi_{n}, \Phi_{n}\right\rangle_{\mathcal{W}}}{\lambda_{n}}$. Now, we introduce the complex Fourier coefficients

$$
a_{n}=\frac{\tilde{e}_{n}-i \tilde{f}_{n}}{2}, \quad a_{-n}=\frac{\tilde{e}_{n}+i \tilde{f}_{n}}{2}, n \in \mathbb{N}^{*}
$$

Whence, from this and the notations of (4.1), the solution $(u, v, z)$ can be written as follows

$$
(u, v, z)=\sum_{n \in \mathbb{Z}^{*}} a_{n} e^{i \sqrt{\lambda_{n}} t} \Phi_{n}
$$


According to Lemma 3.1, it is clear that the set $\left(\bar{\Phi}_{n}\right)_{n \in \mathbb{Z}^{*}}$, with $\bar{\Phi}_{n}=\left(\Phi_{n}, i \sqrt{\lambda_{n}} \Phi_{n}\right)$, forms an orthogonal basis of the energy space $X_{\alpha} \times X_{\alpha-\frac{1}{2}}$. Then from the above, the vector valued solution $U=\left((u, v, z),\left(u_{t}, v_{t}, z_{t}\right)\right)$ of System (1.1)-(1.2)-(1.3) is given by

$$
U(t)=\sum_{n \in \mathbb{Z}^{*}} a_{n} e^{i \sqrt{\lambda_{n}} t} \bar{\Phi}_{n} .
$$

Now we introduce a subspace of the energy space $X_{-\frac{1}{2}} \times X_{-1}$ defined by:

$$
\begin{aligned}
\mathcal{Y} & =\left\{U=\sum_{n \in \mathbb{Z}^{*}} a_{n} \bar{\Phi}_{n} \in X_{-\frac{1}{2}} \times X_{-1}:\|U\|_{\mathcal{Y}}^{2}=\sum_{n \in A} \delta_{n}^{2}\left(\left|\tilde{a}_{n}\right|^{2}+\left|\tilde{a}_{n+1}\right|^{2}\right)+\left|\tilde{a}_{n}+\tilde{a}_{n+1}\right|^{2}\right. \\
& \left.+\sum_{n \in B}\left|\tilde{b}_{n}\right|^{2}<\infty\right\}
\end{aligned}
$$

where $\tilde{a}_{n}=\phi_{n}^{\prime}(1) a_{n}$ and $\tilde{b}_{n}=\phi_{n}^{\prime}(1) b_{n}$. Clearly by Proposition 4.4, $\mathcal{Y}$ is a subspace of the energy space $X_{-\frac{1}{2}} \times X_{-1}=\mathcal{H}_{0} \times \mathcal{H}_{-1}$, where $\mathcal{H}_{0}$ and $\mathcal{H}_{-1}$ are defined by (1.5) and (1.7), respectively. Let us see that System (1.1)-(1.2)-(1.3) is well-posed in $\mathcal{Y}$.

Lemma 4.5 Let $U^{0}=\left(\left(u^{0}, v^{0}, z^{0}\right),\left(u^{1}, v^{1}, z^{1}\right)\right)$ be an element of $\mathcal{Y}$. Then the solution $U$ of (1.1) -(1.2) with initial data $U^{0}$ belongs to $\mathcal{Y}$ for every $t>0$. Furthermore, for any $T>0$ there exists a constant $C(T)>0$ such that

$$
\|U(t)\|_{\mathcal{Y}} \leq C(T)\left\|U^{0}\right\|_{\mathcal{Y}}, \quad 0 \leq t \leq T \text { and } U^{0} \in \mathcal{Y} .
$$

Proof. Given $U^{0} \in \mathcal{Y}$, the unique solution $U$ of (1.1)-(1.2) with initial data $U^{0}$ can be represented in Fourier series as follows

$$
U=\sum_{n \in \mathbb{Z}^{*}} a_{n} \bar{\Phi}_{n}(x) e^{i \sqrt{\lambda_{n}} t}
$$

The Fourier coefficients $a_{n}$ are determined by the initial data $U^{0}=\sum_{n \in \mathbb{Z}^{*}} a_{n} \bar{\Phi}_{n}(x)$. On the other hand,

$$
\begin{aligned}
& \|U(t)\|_{\mathcal{Y}}^{2}=\sum_{n \in A} \delta_{n}^{2}\left(\left|\tilde{a}_{n} e^{i \sqrt{\lambda_{n}} t}\right|^{2}+\left|\tilde{a}_{n+1} e^{i \sqrt{\lambda_{n+1}} t}\right|^{2}\right)+\left|\tilde{a}_{n} e^{i \sqrt{\lambda_{n}} t}+\tilde{a}_{n+1} e^{i \sqrt{\lambda_{n+1}} t}\right|^{2} \\
& +\sum_{n \in B}\left|\tilde{b}_{n} e^{i \sqrt{\lambda_{n}} t}\right|^{2} \leq 2 \sum_{n \in A} \delta_{n}^{2}\left(\left|\tilde{a}_{n}\right|^{2}+\left|\tilde{a}_{n+1}\right|^{2}\right)+\left|\tilde{a}_{n+1}\right|^{2}\left|e^{i \sqrt{\lambda_{n+1}} t}-e^{i \sqrt{\lambda_{n}} t}\right|^{2} \\
& +2 \sum_{n \in A}\left|\tilde{a}_{n}+\tilde{a}_{n+1}\right|^{2}\left|e^{i \sqrt{\lambda_{n}} t}\right|^{2}+\sum_{n \in B}\left|\tilde{b}_{n}\right|^{2} .
\end{aligned}
$$

Since for $n \in A,\left|e^{i \sqrt{\lambda_{n+1}} t}-e^{i \sqrt{\lambda_{n}} t}\right|=\left(\sqrt{\lambda_{n+1}}-\sqrt{\lambda_{n}}\right) t=\delta_{n} t$, then

$$
\begin{aligned}
& \|U(t)\|_{\mathcal{Y}}^{2} \leq C\left(\sum_{n \in A} \delta_{n}^{2}\left(\left|\tilde{a}_{n}\right|^{2}+\left(1+t^{2}\right)\left|\tilde{a}_{n+1}\right|^{2}\right)+\left|\tilde{a}_{n}+\tilde{a}_{n+1}\right|^{2}\right) \\
& +C \sum_{n \in B}\left|\tilde{b}_{n}\right|^{2} \leq C(T)\left\|U^{0}\right\|_{\mathcal{Y}}^{2} .
\end{aligned}
$$


In order to characterize the space $\mathcal{Y}$, we introduce for $n \in A$

$$
q_{n}=\frac{1}{2}\left(\frac{\bar{\Phi}_{n+1}}{\phi_{n+1}^{\prime}(1)}+\frac{\bar{\Phi}_{n}}{\phi_{n}^{\prime}(1)}\right) \text { and } p_{n}=\frac{1}{2 \delta_{n}}\left(\frac{\bar{\Phi}_{n+1}}{\phi_{n+1}^{\prime}(1)}-\frac{\bar{\Phi}_{n}}{\phi_{n}^{\prime}(1)}\right) \text {. }
$$

Proposition 4.6 The set $\left(p_{n}\right)_{n \in A} \cup\left(q_{n}\right)_{n \in A} \cup\left(\frac{\bar{\Phi}_{n}}{\phi_{n}^{\prime}(1)}\right)_{n \in B}$ forms a Riesz basis in the space $\mathcal{Y}$.

Proof. From (4.4), observe that any element $U \in \mathcal{Y}$ can be written as

$$
\begin{aligned}
& U=\sum_{n \in \mathbb{Z}^{*}} a_{n} \bar{\Phi}_{n}=\sum_{n \in A} a_{n} \bar{\Phi}_{n}+a_{n+1} \bar{\Phi}_{n+1}+\sum_{n \in B} b_{n} \bar{\Phi}_{n} \\
& =\sum_{n \in A}\left(\tilde{a}_{n}+\tilde{a}_{n+1}\right) q_{n}+\delta_{n}\left(\tilde{a}_{n+1}-\tilde{a}_{n}\right) p_{n}+\sum_{n \in B} \tilde{b}_{n} \frac{\bar{\Phi}_{n}}{\phi_{n}^{\prime}(1)},
\end{aligned}
$$

where $\tilde{a}_{n}=a_{n} \phi_{n}^{\prime}(1)$ and $\tilde{b}_{n}=b_{n} \phi_{n}^{\prime}(1)$. Whence, the set under consideration is complete in $\mathcal{Y}$. Moreover, if we define on $\mathcal{Y}$ a scalar product such that this set is orthonormal, then the corresponding norm is such that

$$
\|U\|_{*}^{2}=\sum_{n \in A} \delta_{n}^{2}\left|\tilde{a}_{n+1}-\tilde{a}_{n}\right|^{2}+\left|\tilde{a}_{n+1}+\tilde{a}_{n}\right|^{2}+\sum_{n \in B}\left|\tilde{b}_{n}\right|^{2} .
$$

Since $\delta_{n}=\mathcal{O}\left(\frac{1}{n}\right)$, it is easily seen that

$$
\|U\|_{\mathcal{Y}}^{2} \leq \sum_{n \in A} \delta_{n}^{2}\left|\tilde{a}_{n+1}-\tilde{a}_{n}\right|^{2}+\left(1+\delta_{n}^{2}\right)\left|\tilde{a}_{n+1}+\tilde{a}_{n}\right|^{2}+\sum_{n \in B}\left|\tilde{b}_{n}\right|^{2} \leq C\|U\|_{*}^{2} .
$$

On the other hand,

$$
\|U\|_{*}^{2} \leq 2 \sum_{n \in A} \delta_{n}^{2}\left(\left|\tilde{a}_{n}\right|^{2}+\left|\tilde{a}_{n+1}\right|^{2}\right)+\left|\tilde{a}_{n+1}+\tilde{a}_{n}\right|^{2}+\sum_{n \in B}\left|\tilde{b}_{n}\right|^{2} \leq C^{\prime}\|U\|_{\mathcal{Y}}^{2} .
$$

The following theorem provides a precise characterization of the space $\mathcal{Y}$ :

Theorem 4.7 The space $\mathcal{Y}$ coincides algebraically and topologically with the subspace of $\mathcal{H}_{0} \times \mathcal{H}_{-1}$ constituted by the elements $\left(\left(u^{0}, v^{0}, z^{0}\right),\left(u^{1}, v^{1}, z^{1}\right)\right)$ such that $\left(v^{0}, v^{1}\right) \in \mathcal{V}_{2} \times L^{2}(0,1)$, where $\mathcal{V}_{2}$ is defined by (1.6)

In order to prove this theorem, we need some results:

Lemma 4.8 For large $\lambda>0$, we have the following estimate

$$
\left\{\begin{array}{l}
\tilde{v}_{\lambda}(x, \lambda)=a_{2}\left(\rho_{2}(x) \sigma_{2}(x)\right)^{-\frac{1}{4}} \int_{x}^{1} \sqrt{\frac{\rho_{2}(t)}{\sigma_{2}(t)}} d t \frac{\cos \left(\sqrt{\lambda} \int_{x}^{1} \sqrt{\frac{\rho_{2}(t)}{\sigma_{2}(t)}} d t\right)}{2 \lambda}+\mathcal{O}\left(\frac{1}{\lambda^{\frac{3}{2}}}\right), \\
\frac{\partial \tilde{v}_{\lambda}}{\partial x}(x, \lambda)=a_{2}\left(\rho_{2}(x)\right)^{\frac{1}{4}}\left(\sigma_{2}(x)\right)^{-\frac{3}{4}} \int_{x}^{1} \sqrt{\frac{\rho_{2}(t)}{\sigma_{2}(t)}} d t \frac{\sin \left(\sqrt{\lambda} \int_{x}^{1} \sqrt{\frac{\rho_{2}(t)}{\sigma_{2}(t)}} d t\right)}{2 \sqrt{\lambda}}+\mathcal{O}\left(\frac{1}{\lambda}\right) .
\end{array}\right.
$$


Proof. By use of Liouville transformation (e.g., see [17, Chapter 1]) and $w(x)=\int_{x}^{1} \sqrt{\frac{\rho_{2}(t)}{\sigma_{2}(t)}} d t$ together with Lemma 2.1 in [17], the solution $\tilde{v}(x, \lambda)$ of Problem (3.3) satisfies the following integral equation

$$
\begin{aligned}
& \tilde{v}(x, \lambda)=a_{2} \frac{\left(\rho_{2}(x) \sigma_{2}(x)\right)^{-\frac{1}{4}}}{\sqrt{\lambda}} \sin (\sqrt{\lambda} w(x)) \\
& +\frac{\left(\rho_{2}(x) \sigma_{2}(x)\right)^{-\frac{1}{4}}}{\sqrt{\lambda}} \int_{0}^{w(x)} \sin (\sqrt{\lambda}(w(x)-t)) Q(t)\left(\rho_{2}(t) \sigma_{2}(t)\right)^{\frac{1}{4}} \tilde{v}(t, \lambda) d t
\end{aligned}
$$

where $Q(w)=\frac{q_{2}(x)}{\rho_{2}(x)}-\frac{\ddot{\theta}(w)}{\theta(w)}$, with $\theta(w)=\left(\rho_{2}(x) \sigma_{2}(x)\right)^{\frac{1}{4}}$ and $\dot{\theta} \equiv \frac{\partial \theta}{\partial w}$. Differentiating this equation with respect to $\lambda$, we get

$$
\begin{aligned}
& \tilde{v}_{\lambda}(x, \lambda)=a_{2}\left(\rho_{2}(x) \sigma_{2}(x)\right)^{-\frac{1}{4}}\left(w(x) \frac{\cos (\sqrt{\lambda} w(x))}{2 \lambda}+\frac{\sin (\sqrt{\lambda} w(x))}{2 \lambda^{\frac{3}{2}}}\right) \\
& +\frac{\left(\rho_{2}(x) \sigma_{2}(x)\right)^{-\frac{1}{4}}}{2 \lambda} \int_{0}^{w(x)} \sin (\sqrt{\lambda}(w(x)-t)) Q(t)\left(\rho_{2}(t) \sigma_{2}(t)\right)^{\frac{1}{4}}\left(\tilde{v}_{\lambda}(t, \lambda)-\frac{\tilde{v}(t, \lambda)}{\sqrt{\lambda}}\right) d t \\
& +\frac{\left(\rho_{2}(x) \sigma_{2}(x)\right)^{-\frac{1}{4}}}{2 \lambda} \int_{0}^{w(x)}(w(x)-t) \cos (\sqrt{\lambda}(w(x)-t)) Q(t)\left(\rho_{2}(t) \sigma_{2}(t)\right)^{\frac{1}{4}} \tilde{v}(t, \lambda) d t .
\end{aligned}
$$

Let $M(\lambda)=\max _{0 \leq x \leq 1}\left|\tilde{v}_{\lambda}(x, \lambda)\right|$. Using (4.23) and (4.24), one obtains for large $\lambda$, $M(\lambda)\left(1-\frac{\int_{0}^{1}|Q(t)| d t}{\lambda}\right) \leq \frac{C}{\lambda}(c>0)$, and hence

$$
\left|\tilde{v}_{\lambda}(x, \lambda)\right|=\mathcal{O}\left(\frac{1}{\lambda}\right)
$$

By substituting (3.25) and (4.25) into the integrals on the right side of (4.24), the first estimate in (4.22) holds. The second estimate can be obtained in a similar way.

We define the subsets

$$
\begin{gathered}
\Lambda^{*}=\{n \text { and }-n \text { such that } n \in \Lambda\}, \\
=\left\{n \text { and }-n \text { such that } \tilde{u}\left(0, \mu_{n}\right)=\tilde{v}\left(0, \mu_{n}\right)=0\right\}, \\
B^{+}=\left\{n \in B \text { such that } \mu_{n-1} \text { is an eigenvalue of Problem (3.9) }\right\}
\end{gathered}
$$

and

$$
B^{-}=\left\{n \in B \text { such that } \mu_{n-1} \text { is an eigenvalue of Problem (3.8) }\right\},
$$

where the set $\Lambda$ is defined in Proposition 3.8 and $B$ is defined by (4.3). According to (3.43), we have $B=B^{+} \cup B^{-}$.

We establish now the asymptotic behavior of the first derivative of the eigenfunctions $\left(\phi_{n}(x)\right)_{n \in \mathbb{Z}^{*}}$ at $x=1$. 
Proposition 4.9 For every $n \in \mathbb{Z}^{*}, \phi_{n}^{\prime}(1) \neq 0$. Furthermore, for large $n$,

$$
\begin{gathered}
\left|\phi_{n}^{\prime}(1)\right|=\mathcal{O}(1), n \in \Lambda^{*} \cup B^{+}, \\
\left|\phi_{n}^{\prime}(1)\right| \leq \frac{C}{n}, C>0, n \in A \backslash \Lambda^{*}, \\
\left|\phi_{n+1}^{\prime}(1)\right|=\mathcal{O}\left(\frac{1}{n}\right), n \in A
\end{gathered}
$$

and

$$
\left|\phi_{n}^{\prime}(1)\right|=\mathcal{O}\left(\frac{1}{n}\right), n \in B^{-} .
$$

Proof. It is clear from the expressions (3.75) and (3.76) of $\phi_{n}$ together with the initial conditions in (3.18), that

$$
\phi_{n}^{\prime}(1)= \begin{cases}-\sigma_{1}(0) \tilde{u}_{x}\left(0, \lambda_{n}\right), & n \in \Lambda^{*}, \\ -\sqrt{\lambda_{n}} \tilde{u}\left(0, \lambda_{n}\right), & n \in \mathbb{Z}^{*} \backslash \Lambda^{*} .\end{cases}
$$

Using this and (4.12), the estimate (4.29) holds for $n \in \Lambda^{*}$.

In view of (4.15), (4.16) and (4.33), we obtain the estimates (4.30) and (4.31).

Now by (3.10), (3.24) and (3.43), we have

$$
\sqrt{\lambda_{n}} \tilde{u}\left(0, \lambda_{n}\right) \sim \frac{\left(\rho_{1}(0) \sigma_{1}(0)\right)^{-\frac{1}{4}}}{\left(\rho_{1}(-1)\right)^{\frac{1}{4}}\left(\sigma_{1}(-1)\right)^{-\frac{3}{4}}}, \text { for large } n \in B^{+}
$$

and

$$
\sqrt{\lambda_{n}} \tilde{u}\left(0, \lambda_{n}\right)=\mathcal{O}\left(\frac{1}{n}\right), \text { for large } n \in B^{-} .
$$

From this and (4.33), the estimates (4.29) and (4.32) follow.

We are now in position to prove Theorem 4.7. The method used in the following proof was in some parts inspired from the one of Theorem 5.4 in [7].

Proof of Theorem 4.7. To simplify notations, along the proof we denote the norm $\|\cdot\|_{\mathcal{V}_{2} \times L^{2}(0,1)}$ by $\|\cdot\|_{H^{+}}$and we set $w(x)=\int_{x}^{1} \sqrt{\frac{\rho_{2}(t)}{\sigma_{2}(t)}} d t$. First, we consider an element $U \in \mathcal{Y}$ and we prove that $U_{\left.\right|_{(0,1)}} \in \mathcal{V}_{2} \times L^{2}(0,1)$. In view of Proposition 4.6, any element $U \in \mathcal{Y}$ can be written as

$$
U=\sum_{n \in A}\left(c_{n} q_{n}+d_{n} p_{n}\right)+\sum_{n \in B} \tilde{b}_{n} \frac{\bar{\Phi}_{n}}{\phi_{n}^{\prime}(1)}
$$

with the coefficients $\left(\tilde{b}_{n}\right),\left(c_{n}\right)$ and $\left(d_{n}\right)$ are in $\ell^{2}$. Clearly, we have

$$
\sum_{n \in A} c_{n} q_{n}+d_{n} p_{n}=\sum_{n \in A \backslash \Lambda^{*}} c_{n} q_{n}+d_{n} p_{n}+\sum_{n \in \Lambda^{*}} c_{n} q_{n}+d_{n} p_{n}
$$


where the sets $A$ and $\Lambda^{*}$ are defined by (4.2) and (4.26), respectively.

We set $U^{1,1}=\sum_{n \in A \backslash \Lambda^{*}} d_{n} p_{n}$. We prove that $\left\|U^{1,1}\right\|_{H^{+}}<\infty$. From (3.76) and (4.20), one gets for $n \in A \backslash \Lambda^{*}$

$$
\left.p_{n}\right|_{(0,1)}=\frac{1}{2 \delta_{n}}\left(\frac{\sqrt{\lambda_{n+1}} \tilde{u}\left(0, \lambda_{n+1}\right)}{\phi_{n+1}^{\prime}(1)} \overline{\tilde{v}}\left(x, \lambda_{n+1}\right)-\frac{\sqrt{\lambda_{n}} \tilde{u}\left(0, \lambda_{n}\right)}{\phi_{n}^{\prime}(1)} \overline{\tilde{v}}\left(x, \lambda_{n}\right)\right),
$$

where $\bar{f}\left(x, \lambda_{n}\right)=\left(f, i \sqrt{\lambda_{n}} f\right)$. Substituting (4.33) into (4.36), one has

$$
\left.p_{n}\right|_{(0,1)}=-\frac{1}{2 \delta_{n}}\left(\overline{\tilde{v}}\left(x, \lambda_{n+1}\right)-\overline{\tilde{v}}\left(x, \lambda_{n}\right)\right) .
$$

By use of the mean value theorem together with (3.6) and (4.22), we have

$$
\begin{aligned}
\tilde{v}\left(x, \lambda_{n+1}\right)-\tilde{v}\left(x, \lambda_{n}\right) & =\left(\sqrt{\lambda_{n+1}}-\sqrt{\lambda_{n}}\right) \frac{\partial}{\partial \lambda} \tilde{v}\left(x, \lambda^{2}\right)_{\left.\right|_{\lambda=\sqrt{\alpha_{n}}}} \\
& =2 \delta_{n} \sqrt{\alpha_{n}} \tilde{v}_{\lambda}\left(x, \alpha_{n}\right)
\end{aligned}
$$

for some $\sqrt{\alpha_{n}} \in\left(\sqrt{\lambda_{n}}, \sqrt{\lambda_{n+1}}\right)$ and $n \in A$. Using this and (4.37), we obtain

$$
\left.p_{n}\right|_{(0,1)}=-\frac{1}{2 \delta_{n}}\left[2 \delta_{n} \sqrt{\alpha_{n}} \tilde{v}_{\lambda}\left(x, \alpha_{n}\right)\left(1, i \sqrt{\lambda_{n}}\right)+\left(0, i \delta_{n} \tilde{v}\left(x, \lambda_{n+1}\right)\right)\right]
$$

In view of (3.25), it is easily seen that $\left\|\tilde{v}\left(x, \lambda_{n+1}\right)\right\|_{L^{2}(0,1)}^{2}=\mathcal{O}\left(\frac{1}{n^{2}}\right)$. Hence

$$
\begin{aligned}
& \left\|\sum_{n \in A \backslash \Lambda^{*}} \frac{d_{n}}{2 \delta_{n}} \delta_{n} \tilde{v}\left(x, \lambda_{n+1}\right)\right\|_{L^{2}(0,1)}^{2} \leq C\left(\sum_{n \in A \backslash \Lambda^{*}}\left|d_{n}\right|^{2}\right)\left(\sum_{n \in A \backslash \Lambda^{*}}\left\|\tilde{v}\left(x, \lambda_{n+1}\right)\right\|_{L^{2}(0,1)}^{2}\right) \\
& \leq C^{\prime} \sum_{n \in A \backslash \Lambda^{*}}\left|d_{n}\right|^{2}<\infty .
\end{aligned}
$$

Using (4.22), it is clear that $\left\|\sqrt{\alpha_{n}} \overline{\tilde{v}}_{\lambda}\left(x, \alpha_{n}\right)\right\|_{H^{+}}^{2}=\mathcal{O}(1)$. Thus, since $\sqrt{\alpha_{n}}>\sqrt{\lambda_{n}}$, we have

$$
\begin{aligned}
& \left\|\sum_{n \in A \backslash \Lambda^{*}} \frac{d_{n}}{\delta_{n}} \sqrt{\alpha_{n}} \delta_{n} \tilde{v}_{\lambda}\left(x, \alpha_{n}\right)\left(1, i \sqrt{\lambda_{n}}\right)\right\|_{H^{+}}^{2} \leq C\left\|\sum_{n \in A \backslash \Lambda^{*}} \frac{d_{n}}{\delta_{n}} \sqrt{\alpha_{n}} \delta_{n} \bar{v}_{\lambda}\left(x, \alpha_{n}\right)\right\|_{H^{+}}^{2} \\
& \leq C^{\prime} \sum_{n \in A \backslash \Lambda^{*}}\left|d_{n}\right|^{2}+C^{\prime \prime} \sum_{\substack{n, m \in A \backslash \Lambda^{*} \\
n \neq m}}\left|d_{n} d_{m}\left\langle\sqrt{\alpha_{n}} \overline{\tilde{v}}_{\lambda}\left(x, \alpha_{n}\right), \sqrt{\alpha_{m}} \overline{\tilde{v}}_{\lambda}\left(x, \alpha_{m}\right)\right\rangle_{H^{+}}\right| .
\end{aligned}
$$

As noted before, for large $n \in A, \sqrt{\mu_{n-1}}$ close to $\sqrt{\mu_{n}}$, and hence, by Corollary 3.7 ,

$$
\sqrt{\lambda_{n}}-\sqrt{\mu_{n-1}} \leq \frac{C}{n} \text { and } \sqrt{\mu_{n}}-\sqrt{\lambda_{n}} \leq \frac{C}{n}
$$


for some $C>0$. Since $\sqrt{\alpha_{n}} \in\left(\sqrt{\lambda_{n}}, \sqrt{\lambda_{n+1}}\right)$ and $\delta_{n}=\sqrt{\lambda_{n+1}}-\sqrt{\lambda_{n}}=\mathcal{O}\left(\frac{1}{n}\right)$, then

$$
\left|\sqrt{\alpha_{n}}-\sqrt{\mu_{n-1}}\right| \leq \frac{C}{n} \text { and }\left|\sqrt{\alpha_{n}}-\sqrt{\mu_{n}}\right| \leq \frac{C}{n}
$$

Without loss of generality, we may assume that $\mu_{n-1}$ is an eigenvalue of Problem (3.9)). Then by (3.10) $),\left|\sqrt{\alpha_{n}}-\frac{(n-1) \pi}{\gamma_{2}}\right|=\mathcal{O}\left(\frac{1}{n}\right)$. From this and (4.22), we have for large $n$,

$$
\sqrt{\alpha_{n}} \frac{\partial}{\partial x} \tilde{v}_{\lambda}\left(x, \alpha_{n}\right)=\frac{a_{2}}{2}\left(\rho_{2}(x)\right)^{\frac{1}{4}}\left(\sigma_{2}(x)\right)^{-\frac{3}{4}} w(x) \sin \left(\frac{(n-1) \pi}{\gamma_{2}}\right)+\mathcal{O}\left(\frac{1}{n}\right) .
$$

Using this, yields

$$
\begin{aligned}
& \left|\left\langle\sqrt{\alpha_{n}} \tilde{v}_{\lambda}\left(x, \alpha_{n}\right), \sqrt{\alpha_{m}} \tilde{v}_{\lambda}\left(x, \alpha_{m}\right)\right\rangle_{\mathcal{V}_{2}}\right|=\left|\int_{0}^{1} \sqrt{\alpha_{n}} \sqrt{\alpha_{m}} \frac{\partial}{\partial x}\left(\tilde{v}_{\lambda}\right) \frac{\partial}{\partial x}\left(\left(x, \alpha_{n}\right) \tilde{v}_{\lambda}\right)\left(x, \alpha_{m}\right) d x\right| \\
& \leq C\left|\int_{0}^{1}\left(\rho_{2}(x)\right)^{\frac{1}{2}}\left(\sigma_{2}(x)\right)^{-\frac{3}{2}}(w(x))^{2} \sin \left(\frac{(n-1) \pi}{\gamma_{2}} w(x)\right) \sin \left(\frac{(m-1) \pi}{\gamma_{2}} w(x)\right) d x\right| \\
& +\mathcal{O}\left(\frac{1}{|n|}\right)\left|\int_{0}^{1}\left(\rho_{2}(x)\right)^{\frac{1}{4}}\left(\sigma_{2}(x)\right)^{-\frac{3}{4}} w(x) \sin \left(\frac{(m-1) \pi}{\gamma_{2}} w(x)\right) d x\right| \\
& +\mathcal{O}\left(\frac{1}{|m|}\right)\left|\int_{0}^{1}\left(\rho_{2}(x)\right)^{\frac{1}{4}}\left(\sigma_{2}(x)\right)^{-\frac{3}{4}} w(x) \sin \left(\frac{(n-1) \pi}{\gamma_{2}} w(x)\right) d x\right|
\end{aligned}
$$

Integrating by parts, we get

$$
\begin{aligned}
& \mathcal{O}\left(\frac{1}{|n|}\right)\left|\int_{0}^{1}\left(\rho_{2}(x)\right)^{\frac{1}{4}}\left(\sigma_{2}(x)\right)^{-\frac{3}{4}} w(x) \sin \left(\frac{(m-1) \pi}{\gamma_{2}} w(x)\right) d x\right| \\
& \leq \mathcal{O}\left(\frac{1}{|n|}\right) \frac{\left(\rho_{2}(0) \sigma_{2}(0)\right)^{-\frac{1}{4}} \gamma_{2}^{2}}{(m-1) \pi}+\mathcal{O}\left(\frac{1}{|n m|}\right) \leq \frac{C}{|n m|} .
\end{aligned}
$$

Similarly, twice integration by parts, yields

$$
\begin{aligned}
& \left|\int_{0}^{1}\left(\rho_{2}(x)\right)^{\frac{1}{2}}\left(\sigma_{2}(x)\right)^{-\frac{3}{2}}(w(x))^{2} \sin \left(\frac{(n-1) \pi}{\gamma_{2}} w(x)\right) \sin \left(\frac{(m-1) \pi}{\gamma_{2}} w(x)\right) d x\right| \\
& \leq \frac{1}{2}\left|\int_{0}^{1}\left(\rho_{2}(x)\right)^{\frac{1}{2}}\left(\sigma_{2}(x)\right)^{-\frac{3}{2}}(w(x))^{2} \cos \left(\frac{(n-m) \pi}{\gamma_{2}} w(x)\right) d x\right| \\
& +\frac{1}{2}\left|\int_{0}^{1}\left(\rho_{2}(x)\right)^{\frac{1}{2}}\left(\sigma_{2}(x)\right)^{-\frac{3}{2}}(w(x))^{2} \cos \left(\frac{(n+m-2) \pi}{\gamma_{2}} w(x)\right) d x\right| \\
& \leq \frac{\gamma_{2}}{2|n-m| \pi}\left|\int_{0}^{1}\left(\sigma_{2}^{-1}(x)(w(x))^{2}\right)^{\prime} \sin \left(\frac{(n-m) \pi}{\gamma_{2}} w(x)\right) d x\right| \\
& +\frac{\gamma_{2}}{2|n+m-2| \pi}\left|\int_{0}^{1}\left(\sigma_{2}^{-1}(x)(w(x))^{2}\right)^{\prime} \sin \left(\frac{(n+m-2) \pi}{\gamma_{2}} w(x)\right) d x\right| \\
& \leq \frac{C^{\prime}}{(m-n)^{2}}+\frac{C^{\prime \prime}}{(m+n)^{2}} \leq \frac{C}{(m-n)^{2}} .
\end{aligned}
$$


Analogously, we have $\left|\left\langle\alpha_{n} \tilde{v}_{\lambda}\left(x, \alpha_{n}\right), \alpha_{m} \tilde{v}_{\lambda}\left(x, \alpha_{m}\right)\right\rangle_{L^{2}(0,1)}\right| \leq \frac{C^{\prime}}{(n-m)^{2}}$. Hence from the above, in order to prove that the last term in (4.41) is finite, it suffices to show that

$$
\sum_{\substack{n, m \in A \backslash \Lambda^{*} \\ n \neq m}} \frac{\left|d_{n} d_{m}\right|}{(n-m)^{2}}<\infty
$$

Clearly,

$$
\begin{aligned}
& \sum_{\substack{n, m \in A \backslash \Lambda^{*} \\
n \neq m}} \frac{\left|d_{n} d_{m}\right|}{(n-m)^{2}} \leq \sum_{j \neq 0} \frac{1}{j^{2}} \sum_{n \in A \backslash \Lambda^{*}}\left|d_{n} d_{n+j}\right| \\
& \leq \sum_{j \neq 0} \frac{1}{j^{2}} \sum_{n \in A \backslash \Lambda^{*}}\left|d_{n}\right|^{2} \leq C \sum_{n \in A \backslash \Lambda^{*}}\left|d_{n}\right|^{2}<\infty .
\end{aligned}
$$

Therefore,

$$
\sum_{\substack{n, m \in A \backslash \Lambda^{*} \\ n \neq m}}\left|d_{n} d_{m}\left\langle\sqrt{\alpha_{n}} \overline{\tilde{v}}_{\lambda}\left(x, \alpha_{n}\right), \sqrt{\alpha_{m}} \overline{\tilde{v}}_{\lambda}\left(x, \alpha_{m}\right)\right\rangle_{H^{+}}\right| \leq C \sum_{n \in A \backslash \Lambda^{*}}\left|d_{n}\right|^{2}<\infty .
$$

Combining this with (4.40) and (4.41), we conclude that

$$
\left\|U^{1,1}\right\|_{H^{+}}^{2}=\left\|\sum_{n \in A \backslash \Lambda^{*}} d_{n} p_{n}\right\|_{H^{+}}^{2} \leq C \sum_{n \in A \backslash \Lambda^{*}}\left|d_{n}\right|^{2} .
$$

Let $U^{1,2}=\sum_{n \in A \backslash \Lambda^{*}} c_{n} q_{n}$. Then

$$
\left\|U^{1,2}\right\|_{H^{+}}^{2} \leq 2\left\|\sum_{n \in A \backslash \Lambda^{*}} c_{n}\left(q_{n}-\frac{\bar{\Phi}_{n}}{\phi_{n}^{\prime}(1)}\right)\right\|_{H^{+}}^{2}+2\left\|\sum_{n \in A \backslash \Lambda^{*}} c_{n} \frac{\bar{\Phi}_{n}}{\phi_{n}^{\prime}(1)}\right\|_{H^{+}}^{2} .
$$

In view of (4.20) and (4.44), one has

$$
\left\|\sum_{n \in A \backslash \Lambda^{*}} c_{n}\left(q_{n}-\frac{\bar{\Phi}_{n}}{\phi_{n}^{\prime}(1)}\right)\right\|_{H^{+}}^{2}=\left\|\sum_{n \in A \backslash \Lambda^{*}} c_{n} \delta_{n} p_{n}\right\|_{H^{+}}^{2} \leq C^{\prime} \sum_{n \in A \backslash \Lambda^{*}}\left|c_{n}\right|^{2}<\infty .
$$

By (3.76) and (4.33),

$$
\frac{\bar{\Phi}_{n}}{\phi_{n}^{\prime}(1)}=\overline{\tilde{v}}\left(x, \lambda_{n}\right), \quad \text { for } n \in A \backslash \Lambda^{*}
$$

Then analogously to (4.38), using (4.42), we get for some $\sqrt{\beta_{n}} \in\left(\sqrt{\mu_{n-1}}, \sqrt{\lambda_{n}}\right)$,

$$
\tilde{v}\left(x, \lambda_{n}\right)-\tilde{v}\left(x, \mu_{n-1}\right)=2 \xi_{n} \sqrt{\beta_{n}} \tilde{v}_{\lambda}\left(x, \beta_{n}\right),
$$


with $\left|\xi_{n}\right| \leq \frac{C}{n}$ for large $n$. Substituting (4.47) into (4.46), yields

$$
\frac{\bar{\Phi}_{n}}{\phi_{n}^{\prime}(1)}=\overline{\tilde{v}}\left(x, \mu_{n-1}\right)+2 \xi_{n} \sqrt{\beta_{n}} \tilde{v}_{\lambda}\left(x, \beta_{n}\right)\left(1, i \sqrt{\mu_{n-1}}\right)+\left(0, \xi_{n} \tilde{v}\left(x, \lambda_{n}\right)\right) .
$$

By (4.22), $\left\|\tilde{v}_{\lambda}\left(x, \beta_{n}\right)\left(1, i \sqrt{\mu_{n-1}}\right)\right\|_{H^{+}}^{2}=\mathcal{O}\left(\frac{1}{n^{2}}\right)$. Furthermore, under the above assumption about $\mu_{n-1}$, since $\left\{\overline{\tilde{v}}\left(x, \mu_{n}\right) \text { such that } \tilde{v}\left(0, \mu_{n}\right)=0\right\}_{n \in \mathbb{Z}^{*}}$ is an orthogonal subset in $H^{+}$with $\left\|\overline{\tilde{v}}\left(x, \mu_{n}\right)\right\|_{H^{+}}^{2}=\mathcal{O}(1)$, then from (3.25) and (4.48), we have

$$
\begin{aligned}
& \left\|\sum_{n \in A \backslash \Lambda^{*}} c_{n} \frac{\bar{\Phi}_{n}}{\phi_{n}^{\prime}(1)}\right\|_{H^{+}}^{2} \leq C_{1}\left\|\sum_{n \in A \backslash \Lambda^{*}} c_{n} \xi_{n} \tilde{v}\left(x, \lambda_{n}\right)\right\|\left\|_{L^{2}(0,1)}^{2}+C_{2}\right\| \sum_{n \in A \backslash \Lambda^{*}} c_{n} \bar{v}\left(x, \mu_{n-1}\right) \|_{H^{+}}^{2} \\
& +C_{3}\left\|\sum_{n \in A \backslash \Lambda^{*}} c_{n} \tilde{v}_{\lambda}\left(x, \beta_{n}\right)\left(1, i \sqrt{\mu_{n-1}}\right)\right\|_{H^{+}}^{2} \leq C_{1}\left(\sum_{n \in A \backslash \Lambda^{*}}\left|c_{n} \xi_{n}\right|^{2}\right) \sum_{n \in A \backslash \Lambda^{*}}\left\|\tilde{v}\left(x, \lambda_{n}\right)\right\|_{L^{2}(0,1)}^{2} \\
& +C_{2}^{\prime} \sum_{n \in A \backslash \Lambda^{*}}\left|c_{n}\right|^{2}+C_{3}\left(\sum_{n \in A \backslash \Lambda^{*}}\left|c_{n}\right|^{2}\right) \sum_{n \in A \backslash \Lambda^{*}}\left\|\tilde{v}_{\lambda}\left(x, \beta_{n}\right)\left(1, i \sqrt{\mu_{n-1}}\right)\right\|_{H^{+}}^{2} \\
& \leq C^{\prime} \sum_{n \in A \backslash \Lambda^{*}}\left|c_{n}\right|^{2} .
\end{aligned}
$$

Hence, from this and (4.45), it follows that $\left\|U^{1,2}\right\|_{H^{+}}^{2} \leq C \sum_{n \in A \backslash \Lambda^{*}}\left|c_{n}\right|^{2}$. Therefore, $U_{\mid(0,1)}^{1,1}$ and $U_{\mid(0,1)}^{1,2}$ belong to $\mathcal{V}_{2} \times L^{2}(0,1)$.

Let us now take $U^{2,1}=\sum_{n \in \Lambda^{*}} d_{n} p_{n}$. Recall that if $n \in \Lambda^{*}$, then $\lambda_{n}=\mu_{n}$ and $(n+1) \notin \Lambda^{*}$, so that for $n \in \Lambda^{*}, \Phi_{n}$ and $\Phi_{n+1}$ have the forms (3.75) and (3.76), respectively. Proceeding as above, in view of (4.20), (4.33) and (4.38), one gets

$$
\begin{aligned}
& p_{n}=\frac{1}{2 \delta_{n}}\left(\frac{\sqrt{\lambda_{n+1}} \tilde{u}\left(0, \lambda_{n+1}\right)}{\phi_{n+1}^{\prime}(1)} \tilde{v}\left(x, \lambda_{n+1}\right)-\frac{\sigma_{1}(0) \tilde{u}_{x}\left(0, \lambda_{n}\right)}{\phi_{n}^{\prime}(1)} \bar{v}\left(x, \lambda_{n}\right)\right) \\
& =-\frac{1}{2 \delta_{n}}\left(\bar{v}\left(x, \lambda_{n+1}\right)-\overline{\tilde{v}}\left(x, \lambda_{n}\right)\right) \\
& =-\frac{1}{2 \delta_{n}}\left(2 \sqrt{\alpha_{n}} \delta_{n} \tilde{v}_{\lambda}\left(x, \alpha_{n}\right)\left(1, i \sqrt{\lambda_{n}}\right)+\left(0, i \delta_{n} \tilde{v}\left(x, \lambda_{n+1}\right)\right)\right),
\end{aligned}
$$

for some $\sqrt{\alpha_{n}} \in\left(\sqrt{\lambda_{n}}, \sqrt{\lambda_{n+1}}\right)$. In the same way as in (4.40), (4.41) and (4.43), it can be shown that

$$
\left\|U^{2,1}\right\|_{H^{+}}^{2}=\left\|\sum_{n \in \Lambda^{*}} d_{n} p_{n}\right\|_{H^{+}}^{2} \leq C \sum_{n \in \Lambda^{*}}\left|d_{n}\right|^{2}
$$


We set $U^{2,2}=\sum_{n \in \Lambda^{*}} c_{n} q_{n}$. Similarly, we have

$$
\begin{aligned}
& \left\|U^{2,2}\right\|_{H^{+}}^{2} \leq 2\left\|\sum_{n \in \Lambda^{*}} c_{n}\left(q_{n}-\frac{\bar{\Phi}_{n}}{\phi_{n}^{\prime}(1)}\right)\right\|_{H^{+}}^{2}+2\left\|\sum_{n \in \Lambda^{*}} c_{n} \frac{\bar{\Phi}_{n}}{\phi_{n}^{\prime}(1)}\right\|_{H^{+}}^{2} \\
& \leq 2\left\|\sum_{n \in \Lambda^{*}} c_{n} \delta_{n} p_{n}\right\|_{H^{+}}^{2}+2\left\|\sum_{n \in \Lambda^{*}} c_{n} \bar{v}\left(x, \lambda_{n}\right)\right\|_{H^{+}}^{2} \leq C \sum_{n \in \Lambda^{*}}\left|c_{n}\right|^{2} .
\end{aligned}
$$

Therefore, $U_{\mid(0,1)}^{2,1}$ and $U_{\mid(0,1)}^{2,2}$ belong to $\mathcal{V}_{2} \times L^{2}(0,1)$.

Recall that $B=B^{+} \cup B^{-}$and set $U^{3,1}=\sum_{n \in B^{+}} \tilde{b}_{n} \frac{\bar{\Phi}_{n}}{\phi_{n}^{\prime}(1)}$. Analogously to (4.48), using (3.76), (3.43) and (4.33), one has

$$
\frac{\bar{\Phi}_{n}}{\phi_{n}^{\prime}(1)}=\overline{\tilde{v}}\left(x, \mu_{n-1}\right)+2 \mathcal{O}\left(\frac{1}{n}\right) \sqrt{\beta_{n}} \tilde{v}_{\lambda}\left(x, \beta_{n}\right)\left(1, i \sqrt{\mu_{n-1}}\right)+\left(0, \mathcal{O}\left(\frac{1}{n}\right) \tilde{v}\left(x, \lambda_{n}\right)\right)
$$

for some $\sqrt{\beta_{n}} \in\left(\sqrt{\mu_{n-1}}, \sqrt{\lambda_{n}}\right)$. Now, similarly to (4.49), taking into account that $\left\{\overline{\tilde{v}}\left(x, \mu_{n-1}\right)\right\}_{n \in B^{+}}$is an orthogonal subset in $H^{+}$with $\left\|\overline{\tilde{v}}\left(x, \mu_{n-1}\right)\right\|_{H^{+}}^{2}=\mathcal{O}(1)$, we obtain $\left\|U^{3,1}\right\|_{H^{+}}^{2} \leq C \sum_{n \in B^{+}}\left|\tilde{b}_{n}\right|^{2}$. Thus $U_{\mid(0,1)}^{3,1} \in \mathcal{V}_{2} \times L^{2}(0,1)$.

Let us define $U^{3,2}=\sum_{n \in B^{-}} \tilde{b}_{n} \frac{\bar{\Phi}_{n}}{\phi_{n}^{\prime}(1)}$. Recall that for $n \in B^{-}, \mu_{n-1}$ is not an eigenvalue of Problem (3.9), i.e., $\tilde{v}\left(0, \mu_{n-1}\right) \neq 0$ and so $\left\{\overline{\tilde{v}}\left(x, \mu_{n-1}\right)\right\}_{n \in B^{-}}$is not necessarily an orthogonal subset in $H^{+}$. By (3.25), (3.76) ) and (4.33), we have $\left\|\frac{\bar{\Phi}_{n}}{\phi_{n}^{\prime}(1)}\right\|_{H^{+}}=\mathcal{O}(1)$ for $n \in B^{-}$. Hence

$$
\left\|U^{3,2}\right\|_{H^{+}}^{2} \leq C \sum_{n \in B^{-}}\left|\tilde{b}_{n}\right|^{2}+\sum_{\substack{n, m \in B^{-} \\ n \neq m}}\left|\tilde{b}_{n} \tilde{b}_{m}\left\langle\frac{\bar{\Phi}_{n}}{\phi_{n}^{\prime}(1)}, \frac{\bar{\Phi}_{m}}{\phi_{m}^{\prime}(1)}\right\rangle_{H^{+}}\right|
$$

From (4.21) and (4.32), it is easily seen that $\tilde{b}_{n} \sim \frac{b_{n}}{n}$. On the other hand, clearly by (3.74), (4.17) and (4.18), a simple computation gives

$$
b_{n}=\mathcal{O}(1) \text {, for large } n \in B^{-} \text {. }
$$

Since $\left(\tilde{b}_{n}\right) \in \ell^{2}$, then to see that $\sum_{\substack{n, m \in B^{-} \\ n \neq m}}\left|\tilde{b}_{n} \tilde{b}_{m}\left\langle\frac{\bar{\Phi}_{n}}{\phi_{n}^{\prime}(1)}, \frac{\bar{\Phi}_{m}}{\phi_{m}^{\prime}(1)}\right\rangle_{H^{+}}\right|<\infty$, it suffices to show that

$$
\sum_{\substack{n, m \in B^{-} \\ n \neq m}}\left|\frac{1}{n m}\left\langle\frac{\bar{\Phi}_{n}}{\phi_{n}^{\prime}(1)}, \frac{\bar{\Phi}_{m}}{\phi_{m}^{\prime}(1)}\right\rangle_{H^{+}}\right|=\sum_{\substack{n, m \in B^{-} \\ n \neq m}}\left|\frac{1}{n m}\left\langle\overline{\tilde{v}}\left(x, \lambda_{n}\right), \overline{\tilde{v}}\left(x, \lambda_{m}\right)\right\rangle_{H^{+}}\right|<\infty .
$$


Hence, using (3.25) and integrating by parts, we get

$$
\left|\left\langle\overline{\tilde{v}}\left(x, \lambda_{n}\right), \bar{v}\left(x, \lambda_{m}\right)\right\rangle_{H^{+}}\right| \leq \frac{C_{1}}{|n-m|}+\frac{C_{2}}{|n+m|} \leq \frac{C}{|n-m|} .
$$

Now, we show that $\sum_{\substack{n, m \in \mathbb{Z}^{*} \\ n \neq m}} \frac{1}{|n m(n-m)|}<\infty$. For fixed $n, \sum_{\substack{m \in \mathbb{Z}^{*} \\ n \neq m}} \frac{1}{|m n(n-m)|}$ converges. Indeed for large enough $N$ and fixed $n$, we have

$$
\sum_{\substack{m=1 \\ n \neq m}}^{N} \frac{1}{m n(m-n)}=\sum_{m=1}^{n-1} \frac{1}{m n(n-m)}+\sum_{m=n+1}^{N} \frac{1}{m n(m-n)} .
$$

It is clear that

$$
\begin{aligned}
& \sum_{m=n+1}^{N} \frac{1}{m n(m-n)}=\frac{1}{n^{2}}\left(\sum_{m=n+1}^{N} \frac{1}{m-n}-\sum_{m=n+1}^{N} \frac{1}{m}\right), \\
& =\frac{1}{n^{2}}\left(\sum_{j=1}^{N-n} \frac{1}{j}-\sum_{j=n+1}^{N} \frac{1}{j}\right)=\frac{1}{n^{2}}\left(\sum_{j=1}^{n} \frac{1}{j}-\sum_{j=N-n+1}^{N} \frac{1}{j}\right)
\end{aligned}
$$

and $\lim _{N \rightarrow+\infty} \frac{1}{n^{2}}\left(\sum_{j=1}^{n} \frac{1}{j}-\sum_{j=N-n+1}^{N} \frac{1}{j}\right)=\frac{1}{n^{2}} \sum_{j=1}^{n} \frac{1}{j}$. In a similar way, we have

$$
\begin{aligned}
& \sum_{m=1}^{n-1} \frac{1}{m n(n-m)}=\frac{1}{n^{2}}\left(\sum_{m=1}^{n-1} \frac{1}{n-m}+\sum_{m=1}^{n-1} \frac{1}{m}\right) \\
& =\frac{1}{n^{2}}\left(\sum_{j=1}^{n-1} \frac{1}{j}+\sum_{m=1}^{n-1} \frac{1}{m}\right)=\frac{2}{n^{2}} \sum_{j=1}^{n-1} \frac{1}{j} .
\end{aligned}
$$

Since $\sum_{n \in \mathbb{Z}^{*}} \frac{1}{n^{2}}\left(\sum_{j=1}^{n} \frac{1}{j}\right)<\infty$, then the estimate (4.52) follows. From this and (4.50), we have $\left\|U^{3,2}\right\|_{H^{+}}^{2}<\infty$. Therefore, $U_{\mid(0,1)}^{3,2} \in \mathcal{V}_{2} \times L^{2}(0,1)$.

We have proved that if $U \in \mathcal{Y}$, then the restriction of $U$ to $(0,1)$ belongs to $\mathcal{V}_{2} \times L^{2}(0,1)$.

Consider now an element $U=\left(\left(u^{0}, v^{0}, z^{0}\right),\left(u^{1}, v^{1}, z^{1}\right)\right) \in X_{-\frac{1}{2}} \times X_{-1}$ such that $U_{\mid(0,1)} \in \mathcal{V}_{2} \times L^{2}(0,1)$. We shall prove that $U \in \mathcal{Y}$.

Following the proof of Proposition 4.6. $U \in X_{-\frac{1}{2}} \times X_{-1}$ can be written as follows

$$
U=\sum_{n \in \mathbb{Z}^{*}} a_{n} \bar{\Phi}_{n}=\sum_{n \in A} c_{n} q_{n}+d_{n} \delta_{n} p_{n}+\sum_{n \in B} \tilde{b}_{n} \frac{\bar{\Phi}_{n}}{\phi_{n}^{\prime}(1)}
$$


where $c_{n}=\tilde{a}_{n+1}+\tilde{a}_{n}$ and $d_{n}=\tilde{a}_{n+1}-\tilde{a}_{n}$ for $n \in A$. According to Proposition 4.6, to see that $U \in \mathcal{Y}$, it is sufficient to show that $\left(c_{n}\right)_{n \in A} \in \ell^{2},\left(\delta_{n} d_{n}\right)_{n \in A} \in \ell^{2}$ and $\left(\tilde{b}_{n}\right)_{n \in \tilde{\sigma}} \in \ell^{2}$.

Since $\tilde{b}_{n}=b_{n} \phi_{n}^{\prime}(1)$, then by (4.32) and (4.51), we get $\sum_{n \in B^{-}}\left|\tilde{b}_{n}\right|^{2}<\infty$. Proceeding as above, since $\left(\tilde{b}_{n}\right)_{n \in B^{-}} \in \ell^{2}$, then $U_{\mid(0,1)}^{3,2}=\left.\sum_{n \in B^{-}} \tilde{b}_{n} \frac{\bar{\Phi}_{n}}{\phi_{n}^{\prime}(1)}\right|_{(0,1)} \in \mathcal{V}_{2} \times L^{2}(0,1)$. Therefore, $U^{*}=U-U^{3,2}$ is also such that, when restricted to $(0,1)$, belongs to $\mathcal{V}_{2} \times L^{2}(0,1)$. As above, using (3.75) and (3.76), one has

$$
\begin{aligned}
& U_{\mid(0,1)}^{*}=\left(\sum_{n \in \mathbb{Z}^{*} \backslash B} a_{n} \bar{\Phi}_{n}+\sum_{n \in B^{+}} b_{n} \bar{\Phi}_{n}\right)_{\mid(0,1)}=-\sum_{n \in \Lambda^{*}} \tilde{a}_{n} \overline{\tilde{v}}\left(x, \lambda_{n}\right) \\
& -\sum_{n \in A \backslash \Lambda^{*}} \tilde{a}_{n}\left[\left(\overline{\tilde{v}}\left(x, \mu_{n-1}\right)+2 \xi_{n} \sqrt{\alpha_{n}} \tilde{v}_{\lambda}\left(x, \alpha_{n}\right)\left(1, i \sqrt{\mu_{n-1}}\right)\right)+\xi_{n}\left(0, i \tilde{v}\left(x, \lambda_{n}\right)\right)\right] \\
& -\sum_{n \in A}\left[\tilde{a}_{n+1}\left(\overline{\tilde{v}}\left(x, \mu_{n-1}\right)+2 \mathcal{O}\left(\frac{1}{n}\right) \sqrt{\alpha_{n}^{\prime}} \tilde{v}_{\lambda}\left(x, \alpha_{n}^{\prime}\right)\left(1, i \sqrt{\mu_{n-1}}\right)\right)+\mathcal{O}\left(\frac{1}{n}\right)\left(0, i \tilde{v}\left(x, \lambda_{n+1}\right)\right)\right] \\
& -\sum_{n \in B^{+}} \tilde{b}_{n}\left[\left(\overline{\tilde{v}}\left(x, \mu_{n-1}\right)+2 \mathcal{O}\left(\frac{1}{n}\right) \sqrt{\beta_{n}} \tilde{v}_{\lambda}\left(x, \beta_{n}\right)\left(1, i \sqrt{\mu_{n-1}}\right)\right)+\mathcal{O}\left(\frac{1}{n}\right)\left(0, \tilde{v}\left(x, \lambda_{n}\right)\right)\right],
\end{aligned}
$$

for some $\sqrt{\alpha_{n}} \in\left(\sqrt{\mu_{n-1}}, \sqrt{\lambda_{n}}\right)$ for $n \in A \backslash \Lambda^{*}, \sqrt{\alpha_{n}^{\prime}} \in\left(\sqrt{\mu_{n-1}}, \sqrt{\lambda_{n+1}}\right)$ for $n \in A$ and $\sqrt{\beta_{n}} \in\left(\sqrt{\mu_{n-1}}, \sqrt{\lambda_{n}}\right)$ for $n \in B^{+}$. Since $U \in X_{-\frac{1}{2}} \times X_{-1}$, then from (4.6), (4.8) and (4.53), we have

$$
\sum_{n \in A \backslash \Lambda^{*}} \frac{\left|a_{n}\right|^{2}}{\lambda_{n}}\left\langle\Phi_{n}, \Phi_{n}\right\rangle_{\mathcal{W}}<\infty .
$$

From assertion (ii) of Lemma 3.6 and the estimates (4.10) and (4.13) together with (4.30) and (4.33), we obtain

$$
\left\langle\Phi_{n}, \Phi_{n}\right\rangle_{\mathcal{W}} \sim\left|\phi_{n}^{\prime}(1)\right|^{2}, \text { for } n \in A \backslash \Lambda^{*}
$$

As $\tilde{a}_{n}=a_{n} \phi_{n}^{\prime}(1)$, then by (4.55),$\sum_{n \in A \backslash \Lambda^{*}} \frac{\left|\tilde{a}_{n}\right|^{2}}{n^{2}}<\infty$. Since $\left|\xi_{n}\right| \leq \frac{C}{n}$ for large $n$, then

$$
\sum_{n \in A \backslash \Lambda^{*}}\left|\tilde{a}_{n} \xi_{n}\right|^{2}<\infty
$$

Analogously, using (4.7) and (4.9) together with (4.29) and (4.31), we get

$$
\sum_{n \in \Lambda^{*}} \frac{\left|\tilde{a}_{n}\right|^{2}}{n^{2}}<\infty, \sum_{n \in B^{+}} \frac{\left|\tilde{b}_{n}\right|^{2}}{n^{2}}<\infty \text { and } \sum_{n \in A} \frac{\left|\tilde{a}_{n+1}\right|^{2}}{n^{2}}<\infty .
$$


As $\delta_{n}=\mathcal{O}\left(\frac{1}{n}\right)$ for $n \in A$, then from (4.56) and (4.57), we conclude that $\left(\delta_{n} d_{n}\right)_{n \in A} \in \ell^{2}$. In view of (3.25), (4.56) and (4.57),

$$
\begin{gathered}
\left\|\sum_{n \in A \backslash \Lambda^{*}} \tilde{a}_{n} \xi_{n} \tilde{v}\left(x, \lambda_{n}\right)\right\|_{L^{2}(0,1)} \leq C \sum_{n \in A \backslash \Lambda^{*}}\left|\tilde{a}_{n} \xi_{n}\right|^{2}<\infty, \\
\left\|\sum_{n \in A} \tilde{a}_{n+1} \mathcal{O}\left(\frac{1}{n}\right) \tilde{v}\left(x, \lambda_{n+1}\right)\right\|_{L^{2}(0,1)} \leq C \sum_{n \in A}\left|\frac{\tilde{a}_{n+1}}{n}\right|^{2}<\infty
\end{gathered}
$$

and

$$
\left\|\sum_{n \in B^{+}} \tilde{b}_{n} \mathcal{O}\left(\frac{1}{n}\right) \tilde{v}\left(x, \lambda_{n}\right)\right\|_{L^{2}(0,1)} \leq C^{\prime} \sum_{n \in B^{+}}\left|\frac{\tilde{b}_{n}}{n}\right|^{2}<\infty .
$$

In a similar way as in (4.41) and (4.43), by (4.56) and (4.57), yields

$$
\begin{aligned}
& \left\|\sum_{n \in A \backslash \Lambda^{*}} \tilde{a}_{n} \xi_{n} \sqrt{\alpha_{n}} \tilde{v}_{\lambda}\left(x, \alpha_{n}\right)\left(1, i \sqrt{\mu_{n-1}}\right)\right\|_{H^{+}} \leq C_{1} \sum_{n \in A \backslash \Lambda^{*}}\left|\tilde{a}_{n} \xi_{n}\right|^{2}<\infty, \\
& \left\|\sum_{n \in A} \tilde{a}_{n+1} \mathcal{O}\left(\frac{1}{n}\right) \sqrt{\alpha_{n}^{\prime}} \tilde{v}_{\lambda}\left(x, \alpha_{n}^{\prime}\right)\left(1, i \sqrt{\mu_{n-1}}\right)\right\|_{H^{+}} \leq C_{2} \sum_{n \in A}\left|\frac{\tilde{a}_{n+1}}{n}\right|^{2}<\infty
\end{aligned}
$$

and

$$
\left\|\sum_{n \in B^{+}} \tilde{b}_{n} \mathcal{O}\left(\frac{1}{n}\right) \sqrt{\beta_{n}} \tilde{v}_{\lambda}\left(x, \beta_{n}\right)\left(1, i \sqrt{\mu_{n-1}}\right)\right\|_{H^{+}} \leq C_{3} \sum_{n \in B^{+}}\left|\frac{\tilde{b}_{n}}{n}\right|^{2}<\infty .
$$

As $U_{(0,1)}^{*} \in \mathcal{V}_{2} \times L^{2}(0,1)$, then from the above and (4.54), we have

$$
\left\|\sum_{n \in \Lambda^{*}} \tilde{a}_{n} \overline{\tilde{v}}\left(x, \lambda_{n}\right)+\sum_{n \in \mathbb{Z}^{*} \backslash\left(\Lambda^{*} \cup B\right)} \tilde{a}_{n} \overline{\tilde{v}}\left(x, \mu_{n-1}\right)+\sum_{n \in B^{+}} \tilde{b}_{n} \overline{\tilde{v}}\left(x, \mu_{n-1}\right)\right\|_{H^{+}}<\infty .
$$

Recall that $\lambda_{n}=\mu_{n}$ for $n \in \Lambda^{*}$ and $\left\{\overline{\tilde{v}}\left(x, \mu_{n}\right) \text { such that } \tilde{v}\left(0, \mu_{n}\right)=0\right\}_{n \in \mathbb{Z}^{*}}$ is an orthogonal subset in $\mathcal{V}_{2} \times L^{2}(0,1)$ with $\left\|\overline{\tilde{v}}\left(x, \mu_{n}\right)\right\|_{H^{+}}=\mathcal{O}(1)$. Under the above assumption, since $\mu_{n-1}$ is an eigenvalue of Problem (3.9), then in view of (4.58), we obtain

$$
\sum_{n \in \Lambda^{*}}\left|\tilde{a}_{n}\right|^{2}<\infty, \sum_{n \in A \backslash \Lambda^{*}}\left|\tilde{a}_{n}\right|^{2}<\infty, \sum_{n \in A}\left|\tilde{a}_{n+1}\right|^{2}<\infty \text { and } \sum_{n \in B^{+}}\left|\tilde{b}_{n}\right|^{2}<\infty .
$$

This implies that $\left(c_{n}\right)_{n \in A} \in \ell^{2}$ and $\left(\tilde{b}_{n}\right)_{n \in B^{+}} \in \ell^{2}$. The proof is complete now. 


\section{Proof Of Theorem 1.2}

In this section we prove the main controllability result for System (1.1)-(1.4). Notice that, in view of the fact that (1.1)-(1.4) is linear and reversible in time, this system is exactly controllable if and only if the system is null controllable. Using HUM [18], the controllability of System (1.1)-(1.4) can be reduced to the obtention of an observability inequality (1.8) of the uncontrolled System (1.1)-(1.2). As a direct application of Lemma 4.2, for $T>2 \gamma$ there exists a positive constant $C$ such that

$$
\begin{aligned}
& \int_{0}^{1}\left|v_{x}(1, t)\right|^{2} d t=\int_{0}^{1} \sum_{n \in \mathbb{Z}^{*}}\left|a_{n} e^{i \sqrt{\lambda_{n}} t} \phi_{n}^{\prime}(1)\right|^{2} d t \\
& \geq C \sum_{n \in A}\left(\sqrt{\lambda_{n+1}}-\sqrt{\lambda_{n}}\right)\left(\left|a_{n} \phi_{n}^{\prime}(1)\right|^{2}+\left|a_{n+1} \phi_{n+1}^{\prime}(1)\right|^{2}\right) \\
& +C \sum_{n \in A}\left|a_{n+1} \phi_{n+1}^{\prime}(1)+a_{n} \phi_{n}^{\prime}(1)\right|^{2}+\sum_{n \in B}\left|b_{n} \phi_{n}^{\prime}(1)\right|^{2} .
\end{aligned}
$$

By setting $\tilde{a}_{n}=a_{n} \phi_{n}^{\prime}(1)$, we obtain

$$
\begin{aligned}
& \int_{0}^{1}\left|v_{x}(1, t)\right|^{2} d t \geq C\left(\sum_{n \in A} \delta_{n}^{2}\left(\left|\tilde{a}_{n}\right|^{2}+\left|\tilde{a}_{n+1}\right|^{2}\right)+\left|\tilde{a}_{n+1}+\tilde{a}_{n}\right|^{2}\right) \\
& +C \sum_{n \in B}\left|\tilde{b}_{n}\right|^{2}=C\left\|U^{0}\right\|_{\mathcal{Y}}^{2}
\end{aligned}
$$

with $U^{0}=\left(\left(u^{0}, v^{0}, z^{0}\right),\left(u^{1}, v^{1}, z^{1}\right)\right)$ and the asymmetric space $\mathcal{Y}$ is defined by (4.19) and characterized by Theorem 4.7. The second inequality of (1.8) can be proved in a similar way. This ends up the proof of Theorem 1.2 .

Remark 5.1 Notice that if the coefficients $\rho_{i}, \sigma_{i}, q_{i} i=1,2$, are symmetric functions, then the eigenvalues $\mu_{n}^{-}$and $\mu_{n}^{+}$of Problems (3.8) and (3.9) coincide. Moreover, we have $\lambda_{\varphi(n)}=\lambda_{2 n}=\mu_{n}^{+}, A=\left\{2 n, n \in \mathbb{Z}^{*}\right\}$ and $B=\emptyset$. Hence, we can use Ullrich's theorem [22], as in [7, 14], to prove the exact controllability.

Remark 5.2 In the case of Neumann boundary control, (i.e., $v_{x}(1, t)=p(t)$ and $u(-1, t)=0)$, we show that the associated spectral gap $\left(\sqrt{\lambda_{n+1}}-\sqrt{\lambda_{n}}\right)$ is uniformly positive if and only if $\frac{\gamma_{1}}{\gamma}$ is a rational number with an irreductible fraction such that $\frac{\gamma_{1}}{\gamma}=\frac{2 p-1}{q}$, where $\gamma_{1}=\int_{-1}^{0}\left(\frac{\rho(x)}{\sigma(x)}\right)^{\frac{1}{2}} d x, \gamma=\int_{-1}^{0}\left(\frac{\rho_{1}(x)}{\sigma_{1}(x)}\right)^{\frac{1}{2}} d x+\int_{0}^{1}\left(\frac{\rho_{2}(x)}{\sigma_{2}(x)}\right)^{\frac{1}{2}} d x$ and $p, q \in$ $\mathbb{N}^{*}$. Under this condition, we prove that this system is exactly controllable in an asymmetric space for a control time $T>2 \gamma$. This will be the object of a subsequent paper.

Remark 5.3 Note that the method used in this paper could be extended to prove the exact boundary controllability of hinged two Euler-Bernoulli beams connected by a point mass with variable coefficients. The case of constant coefficients was studied in 
[8]. In that paper, it was shown that the associated spectral gap is uniformly positive, so that by use of Haraux's theorem, it was proved the exact controllability. It could be conjectured that the results obtained in [8] remain true in the case of variable coefficients: all the eigenvalues are simple, the associated spectral gap is uniformly positive, the first derivative of all the eigenfunctions do not vanish at the extreme $x=1$.

\section{References}

[1] F. V. Atkinson and A. B. Mingarelli, Asymptotics of the number of zeros and of the eigenvalues of general weighted Sturm-Liouville problems, Reine Angew. Math., 375(6) (1987), 380-393.

[2] S. Avdonin and J. Edward, Exact controllability for string with attached masses, SIAM J. Cont. Optim., to appear.

[3] S. Avdonin and S. Ivanov, Exponential Riesz bases of subspaces and divided differences, St. Petersburg Mathematical Journal, 13 (2001), no. 3, 339-351.

[4] S. Avdonin and W. Moran, Ingham type inequalities and Riesz bases of divided differences, International Journal of Applied Math. and Computer Science, 11 (2001), no. 4, 101-118.

[5] C. Baiocchi, V. Komornik and P. Loreti, Ingham-Beurling type theorems with weakened gap conditions, Acta Math. Hungar., 97 (1-2) (2002), 55-95.

[6] J. Ben Amara and A.A. Shkalikov, A Sturm-Liouville problem with physical and spectral parameters in boundary conditions, Math. Notes, 66 (2) (1999), 163-172.

[7] C. Castro, Asymptotic analysis and control of a hybrid system composed by two vibrating strings connected by a point mass, ESAIM Control Optim. Calc. Var., 2 (1997), 231-280.

[8] C. Castro and E. Zuazua, Exact boundary controllability of two Euler-Bernoulli beams connected by a point mass, Math. Comput. Modelling, 32 (2000), 955-969.

[9] C. Castro and E. Zuazua, Boundary controllability of hybrid system consisting in two flexible beams connected by a point mass, SIAM J. Cont. Optim., 36 (5) (1998), 1576-1595.

[10] C. Castro and E. Zuazua, A hydrid system consisting of two flexible beams connected by a point mass: Spectral analysis and well-posedness in asymmetric spaces, ESAIM Proceedings, (1997), 17-53. 
[11] J. Eckhardt, An inverse spectral problem for a star graph of Krein strings, preprint posted in Institut Mittag-Leffler, REPORT No. 3, 2012/2013, Spring, to be published in J. Reine Angew. Math.

[12] M. V. Fedoryuk, Asymptotic Analysis, Springer-Verlag, (1983).

[13] I. Ts. Gohberg and M. G. Krein, Theory and appllications of Volterra Operators in Hilbert space, Transl. Math. Mon. 24, Providence, (1970).

[14] S. Hansen and E. Zuazua, Exact controllability and stabilization of a vibrating string with an interior point mass, SIAM J. Cont. Optim., 33 (5) (1995), 13571391.

[15] S. Hansen and J. J. Martinez, Null boundary controllability of a 1-dimensional heat equation with an internal point mass, IEEE 55th Conference on Decision and Control, (2016), 4803-4808.

[16] A. Hurwitz and R. Courant, Theory of functions, Springer Verlag, Berlin, (1964).

[17] B. M. Levitan and I. S. Sargsjan, Introduction to spectral theory: Selfadjoint Ordinary Differential Operators, Americain Mathematical Society, Translation of Mathematical Monographs Vol 39, (1975).

[18] J. L. Lions, Contrôlabilité exacte, perturbations et stabilisation de systèmes distribués, Tome 1, Contrôlabilité exacte, Masson, RMA, Paris, (1988).

[19] D. Mercier and V. Rgnier, Spectrum of a network of Euler-Bernoulli beams, J. Math. Anal. Appl, 337 (2008), 174-196.

[20] D. Mercier and V. Rgnier, Control of a network of Euler-Bernoulli beams, J. Math. Anal. Appl., 342 (2008), 874-894.

[21] H. Mounier, J. Rudolph, M. Fliess and P. Rouchon, Tracking control of a vibrating string with an interior mass viewed as delay system, ESAIM Control Optim. Calc. Var., 3 (1998), 315-321.

[22] D. Ullrich, Divided differences and systems of nonharmonic Fourier series, Proc. AMS, 80(1) (1980), 47-57. 